\title{
Potential Scour at Bridge A07011, Over the Powwow River at Pond Street in Amesbury, Massachusetts
}

\section{By PETER J. MURPHY and LISA BRATTON}

U.S. Geological Survey

Open-File Report 97-801 


\title{
U.S. DEPARTMENT OF THE INTERIOR BRUCE BABBITT, Secretary
}

\author{
U.S. GEOLOGICAL SURVEY \\ Mark Schaefer, Acting Director
}

The use of trade or product names in this report is for identification purposes only and does not constitute endorsement by the U.S. Geological Survey.

Chief, Massachusetts-Rhode Island District U.S. Geological Survey Water Resources Division 28 Lord Road, Suite 280 Marlborough, MA 01752
U.S. Geological Survey Branch of Information Services Box 25286, Federal Center Denver, CO 80225-0286 


\section{CONTENTS}

Summary

Introduction

Overview of Bridge Site

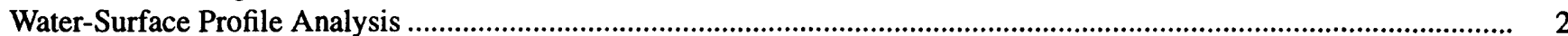

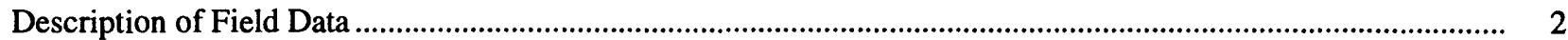

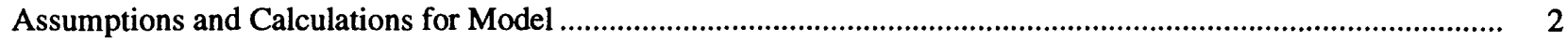

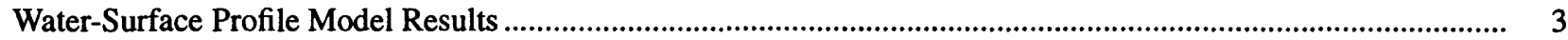

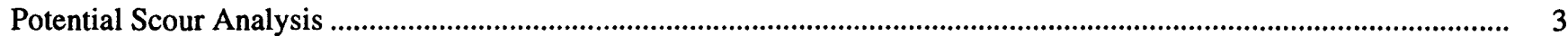

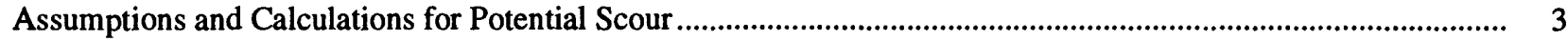

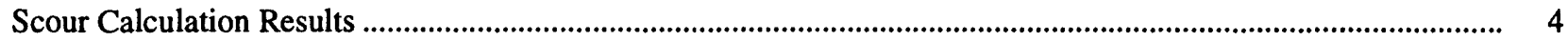

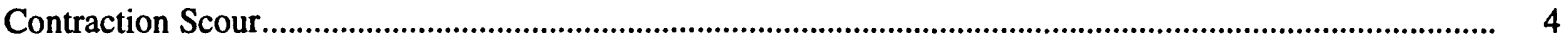

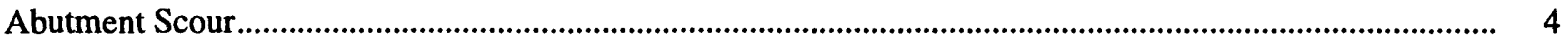

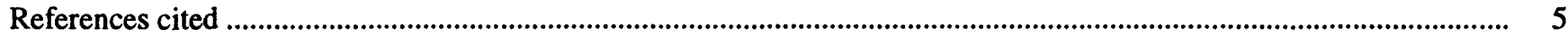

Appendixes

A. WSPRO Input Data File for Bridge A07011, Amesbury, Massachusetts ................................................... 15

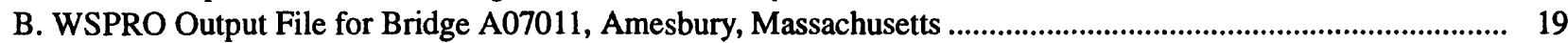

\section{FIGURES}

1. Cross section showing riverbed and flood water level profiles for bridge A07011, over the Powwow River at Pond Street in Amesbury, Massachusetts

2. Cross section at the downstream face of bridge A07011, showing the low chord, water surface

for the maximum discharge before pressure flow, riverbed and potential contraction scour

\section{TABLES}

1. Summary of potential scour depths for bridge A07011, over the Powwow River at Pond Street in Amesbury, Massachusetts

2. Determination of live-bed or clear water conditions for bridge A07011, over the Powwow River at Pond Street in Amesbury, Massachusetts

3. Calculation of clear water contraction scour for bridge A07011, over the Powwow River at

Pond Street in Amesbury, Massachusetts

4. Calculation of local scour at the left abutment for bridge A07011, over the Powwow River at Pond Street in Amesbury, Massachusetts

5. Calculation of local scour at the right abutment for bridge A07011, over the Powwow River at Pond Street in Amesbury, Massachusetts .

\section{CONVERSION FACTORS}

\begin{tabular}{rll}
\hline Multiply & By & To obtain \\
\hline inch (in) & 25.4 & millimeter $(\mathrm{mm})$ \\
foot $(\mathrm{ft})$ & 0.3048 & meter \\
foot per mile $(\mathrm{ft} / \mathrm{mi})$ & 0.1894 & meter per kilometer \\
$\mathrm{mile}(\mathrm{mi})$ & 1.609 & kilometer \\
square $\mathrm{mile}^{\left(\mathrm{mi}^{2}\right)}$ & 2.590 & square kilometer \\
foot per second $(\mathrm{ft} / \mathrm{s})$ & 0.3048 & meter per second \\
cubic foot per second $(\mathrm{ft} / \mathrm{s})$ & 0.02832 & cubic meter per second \\
\hline
\end{tabular}




\title{
Potential Scour at Bridge A07011, Over the Powwow River at Pond Street in Amesbury, Massachusetts
}

\author{
By Peter J. Murphy and Lisa Bratton
}

\section{SUMMARY}

An analysis for potential contraction, abutment, and pier scour was completed for bridge A07011 over the Powwow River at Pond Street in Amesbury, Massachusetts. This report is one of a series completed for selected bridge sites in Massachusetts. The study of scour at this and the other bridges was conducted by the U.S.

Geological Survey in cooperation with the Massachusetts Highway Department.

The bridge is a single arch, stone masonry structure, 33 feet wide and 25 feet long, with no piers and small wingwalls. The land use near the bridge is mainly urban. The streambed material is predominately gravel ( $d_{50}$ is 0.035 foot). Cross sections were surveyed upstream and downstream from the bridge site, at a dam downstream from the bridge, along the roadway, and at both the downstream and upstream bridge-face openings. The backwater curves for four flood flow rates, $Q_{10}, Q_{50}, Q_{100}$, and $Q_{500}$, corresponding to 10 -, $50-, 100-$, and 500 -year return periods, and also for the maximum discharge before pressure flow under the bridge and road overflow, were calculated using a water-surface-profile analysis. The flood discharges ranged from 921 to 3,630 cubic feet per second. The water-surface-profile analysis of the bridge hydraulics showed high velocities (5.52 to 11.9 feet per second) and indicated a potential for scour at this bridge. Table 1 shows potential contraction and abutment scour depths rounded to the nearest foot. The potential contraction and abutment scour are both significant processes.

\section{INTRODUCTION}

The objective of scour depth analysis is to assess and evaluate the stream stability and scour depth at bridge sites. This is one of a series of reports presenting the analysis of scour depths for designated bridges throughout Massachusetts in partial fulfillment of a cooperative agreement between the Massachusetts Highway Department (MHD) and the U.S. Geological Survey (USGS). Each analysis includes a survey of cross sections upstream and downstream of a selected bridge and a survey of the bridge face opening. The survey data were processed and used in a WaterSurface-PROfile (WSPRO) computer model (Shearman, 1990) to determine surface-water levels for four flow rates, $Q_{10}, Q_{50}, Q_{100}$, and $Q_{500}$ for the 10 -, $50-, 100$-, and 500-year floods and for the maximum discharge before pressure flow under the bridge. The results of each computer model were used in scour equations to estimate maximum potential scour depths at the bridge site from contraction, abutment, and pier scour (Richardson and Davis, 1995).

\section{OVERVIEW OF BRIDGE SITE}

The bridge A07011, is over the Powwow River at Pond Street in Amesbury, Massachusetts. The Powwow River is located in the Merrimack River major drainage basin. The bridge is located in MHD District 4 . The bridge is a single arch, stone masonry structure, $33 \mathrm{ft}$ wide and $25 \mathrm{ft}$ long, with no piers and small wingwalls. The drainage area for the site is $50.44 \mathrm{mi}^{2}$. The Powwow River at the bridge site has a channel slope of approximately $0.0002 \mathrm{ft} / \mathrm{ft}(1 \mathrm{ft} / \mathrm{mi})$, an average channel top width of approximately $40 \mathrm{ft}$ and an average channel depth of $10 \mathrm{ft}$ at the 100 -year flood. The predominant streambed material is gravel ( $d_{50}$ is $0.035 \mathrm{ft}$ or $10.6 \mathrm{~mm}$ ). The banks are gravel with 
some silt and sand. The river is regulated at the Wooden Dam, a low dam with a sluice gate, located $262 \mathrm{ft}$ downstream from the bridge.

The land use near the bridge is largely urban. The area on the upstream left side is a grass-covered open area with a house. The words "left" and "right" in this report refer to directions that would be reported by an observer facing downstream. The downstream left side is an old mill building running along the stream to the dam. The upstream right side of the bridge is a grass-covered open area with a house and the downstream right side is a grass-covered area with a sidewalk and a parking lot set back from the stream.

\section{WATER-SURFACE PROFILE ANALYSIS}

The Water-Surface Profile (WSPRO) computer model determines water-surface levels based on backwater calculations. The WSPRO analyses assume a fixed bed and a one-dimensional, gradually varied, and steady flow. The model has several options and can determine overall hydraulic conditions at a site or can approximate transverse distributions of downstream velocity for a predetermined discharge and surface-water level by dividing the channel width into 20 equal-conveyance streamtubes. The computer model uses special routines to compute hydraulic conditions in the vicinity of bridges (Shearman and others, 1986; Shearman, 1990).

A WSPRO model was used at bridge site A07011 to determine the water-surface profile through the bridge opening for four flood flow rates, $Q_{10}, Q_{50}$, $Q_{100}$, and $Q_{500}$. The three smaller floods passed under the bridge without causing pressure flow or road overtopping, but pressure flow and road overtopping occurred (at the same flow rate) before the 500-year flood was attained.

\section{Description of Field Data}

Cross sections were surveyed for the approach (APPR1), roadway (RDWAY), downstream bridge face (BRIDG), exit (EXIT1 and EXIT2), and downstream dam (DSDAM) sections. The dam's spillway acts as a control, approximated as critical depth over a broadcrested weir. The EXIT 2 cross section was located at the upstream face of the dam. The DSDAM cross section was located at the spillway crest. The bridge cross section (BRIDG) was measured at the downstream side of the bridge. The altitude, $497.14 \mathrm{ft}$, of the top of the arch was used as a local datum. The roadway cross section (RDWAY) was surveyed to anticipate potential overtopping of the bridge by a flood.

The streambed was predominately gravel with some sand and underlying bedrock. Manning's roughness values were determined and a scoop sampler was used for collection of medium- and fine-grained material in the riverbed (Hayes, 1993) at the site when the cross sections were surveyed.

\section{Assumptions and Calculations for Model}

Several calculations and assumptions were made before the water-surface model was run:

(1) The flood discharge values for the $Q_{10}, Q_{50}$, $Q_{100}$, and $Q_{500}$ were calculated based on relative drainage basin elevation and drainage basin area, using regression equations developed by P.J. Murphy (U.S. Geological Survey, written commun., 1996). The Flood Insurance Study (FIS) for Amesbury (Federal Emergency Management Agency, 1992) based its flood-flow-rate estimate for the 100-year flood on regression equations developed by Wandle (1983), but did not estimate the size of other floods. The FIS estimate for the 100 -year flood was 13 percent smaller than the Murphy estimate.

(2) One cross section was templated in this analysis; the full-valley (FULLV) section was developed from the EXIT1 section. The section reference distance (SRD) was set to zero at the downstream face of the bridge. The input file for the WSPRO water-surface analysis (Shearman, 1990) is shown in appendix $\mathrm{A}$.

(3) The critical depth of the water at the spillway crest of the Wooden Dam was used to estimate the starting-water-surface elevation downstream from the bridge for the water-surface-profile computations. The FIS for Amesbury (Federal Emergency Management Agency, 1992) used the tidal elevations of the Merrimack River as the starting-water-surface elevations for the Powwow River in Amesbury.

(4) Survey data were processed for input into WSPRO using an Automated WSPRO Input and Survey Processing Program (AWISPP) (E. Boehmler, U.S. Geological Survey, written commun., 1996). AWISPP calculates many of the parameters required in WSPRO such as section-reference distances, and the 
geometry of the bridge, wingwall, abutments, and embankments. AWISPP also was used to calculate channel slope, align cross sections to the left edge of water, process bends in cross section lines, compute the best fit segment line to straighten cross sections, and compute skew angles. The input file for WSPRO created with AWISPP is shown in appendix A.

(5) The left edge of water at the approach, bridge, full valley, and exit cross sections was set to zero to maintain consistency between the sections. This was done in AWISPP by setting the $x$-coordinate of the left edge of water at each station equal to zero.

(6) Because the bridge had vertical abutments, small wingwalls, and a vertical road embankment, the bridge was classified for WSPRO as a type 1A bridge (Shearman, 1990).

(7) The particle-size distribution for the sand and gravel collected at the downstream bridge face was determined using sieve analysis (Folk, 1980). The $d_{50}$ under the bridge is $0.035 \mathrm{ft}$. This grain-size distribution was assumed to apply to the bed material at the approach and exit sections.

(8) The Manning's roughness coefficients for the various parts of the cross sections at the site were assigned values dependent on the bed grain size and on the channel's and overbanks' shapes and roughnesses (Arcement and Schneider, 1984). The stream channel was assigned a value of 0.035 for the whole length of the site. The Manning's roughness coefficient was designated as 0.060 for all the overbank areas. This overbank value includes the effects of the mill building and houses. A wall was set in the dam cross section to show the location of the mill building on the left side of the exit cross section. The FIS for Amesbury (Federal Emergency Management Agency, 1992) used the same roughness coefficients as were used in this study.

\section{Water-Surface Profile Model Results}

The backwater curves for the four flood flow rates, $Q_{10}, Q_{50}, Q_{100}$, and $Q_{500}$, and for the maximum discharge before pressure flow under the bridge were calculated using WSPRO analysis. The flow at the exit cross section was subcritical for all five floods because the Wooden Dam, downstream from the bridge, regulates the water levels at the downstream end of the bridge site.
The computer model calculations indicated the water surface reached low chord of the bridge, pressure flow, and road overtopping at approximately $2,160 \mathrm{ft}^{3} / \mathrm{s}$. The pressure flow occurred before the 500-year flood discharge, but the 500-year flood was included in the water-surface-profile analysis. The results of the computer model calculations are included in appendix B. The WSPRO analysis of the bridge hydraulics showed high velocities at the bridge (5.52 to $11.9 \mathrm{ft} / \mathrm{s})$. The water-surface profiles for the 10-, 50-, 100-, and 500-year floods and for the maximum discharge before pressure flow and road overtopping are shown in figure 1 (at back of report). The FIS for Amesbury showed three water-surface profiles near the bridge at Pond Street that were roughly $1 \mathrm{ft}$ lower than the results of this study. However, the FIS profile for the 500-year flood did not indicate pressure flow or road overtopping and was $3 \mathrm{ft}$ lower than the result of this study.

\section{POTENTIAL SCOUR ANALYSIS}

Scour depths were computed using the general guidelines described in Richardson and Davis (1995) and Arneson and others (1992). The hydraulic model WSPRO was used to determine water-surface profiles and other hydraulic variables needed for scour calculations, such as discharge, velocity, and depth.

\section{Assumptions and Calculations for Potential Scour}

Several equations that are presented and explained in Richardson and Davis (1995) were used to calculate the potential contraction and abutment scour for this bridge, depending on the situation during each flood event. The Neill equation was used to determine the applicability of the live-bed or clear water equations for potential contraction scour. Based on the results of the Neill equation analysis, the appropriate scour equations were used to determine scour depths for the main channel, left overbank, and right overbank of the approach and bridge sections. The contraction scour depths were determined using the Larsen clearwater contraction scour equation. The abutment scour depths were calculated using the Froehlich equation. This report focuses on contraction and abutment scour because the bridge has no piers. 
The HIRE equation (Richardson and Davis, 1995) was not applied to bridges in this study. Although HEC-18 recommended use of the HIRE equation for long $\left(L_{E} / Y_{E}>25\right)$ embankments blocking flow on flood plains, "where conditions are similar to the field conditions from which the equation was derived," those field conditions did not occur at this bridge. The decision to not use the HIRE equation also was recommended in a discussion with L. Arneson, Regional Engineer, FHWA, Denver on January 8, 1997.

Scour depths were calculated assuming an infinite depth of erosive material and a homogeneous particle-size distribution. However, bedrock may underlie the observable channel bed and limit the scour depths.

To clarify the use of variables, different sections of the river reach at the site have been assigned letters associated with the parameters used for the scour calculations from WSPRO output. Variables associated with the approach section are assigned the letter $a$, the variables for the upstream bridge face have a letter $b$, and the downstream bridge face have the letter $c$, with subscripts $m, l$, and $r$ corresponding to the main channel, left flood plain and right flood plain, respectively (tables 2 through 5, at back of report).

\section{Scour Calculation Results}

Scour calculations were done for contraction and abutment scour. The results of the scour depth analysis are presented in tables 3 through 5 . The numbers in the tables have been rounded to 3 significant figures unless otherwise written. The scour depths have been rounded to the nearest foot.

Exposed abutment footings and scour holes were not observed during the field inspection. Riprap was not observed near the bridge foundations, but was observed along both banks just upstream from the bridge and extending along the upstream right bank.

The Neill equation was applied at the approach cross section of the Pond Street bridge over the Powwow River in Amesbury. All flood flows filled the main channel and extended onto both flood plains at the approach cross section. The stream channel under the bridge has no left or right overbanks. The results of the analysis with the Neill equation (table 2) showed that the gravel in the main channel of the approach cross section were too large for sediment motion, thus a clear-water scour analysis was applicable at this bridge.

\section{Contraction Scour}

Laursen's clear-water contraction-scour equation (Richardson and Davis, 1995) was applied to the main channel at the bridge cross section. The calculations are shown in table 3 . The contraction scour results indicated that the $d_{50}$ of the gravel was small enough that clear-water scour would occur at the bridge site for all but the 10-year flood. The contraction scour depths were small, 0 to $6 \mathrm{ft}$. The altitudes of the bottoms of the potential contraction scour holes for the four floods without pressure flow, referenced to the low chord of the bridge at $497.5 \mathrm{ft}$, are $486,483,481$, and $480 \mathrm{ft}$. The altitude of the potential contraction scour for the maximum discharge before pressure flow is shown in figure 2 (at back of report).

\section{Abutment Scour}

Froehlich's abutment scour equation (Richardson and Davis, 1995) was applied to the left and right abutments. Those abutment scour calculations are given in tables 4 and 5. The results show that the right abutment has a range of scour depths from 4 to $7 \mathrm{ft}$ and the left abutment has larger scour depths, from 7 to $12 \mathrm{ft}$. However, no scour was observed on a visit to the site on October 20, 1994 (M. Lombardo, Environmental Careers Organization, written commun., 1994). The depths of the abutment scour are added to the contraction scour depths to determine total scour at the abutments. The altitudes of the bottoms of the total potential scour holes at the left abutment, referenced to the low chord of the bridge at $497.5 \mathrm{ft}$, are $482,480,479$, and $479 \mathrm{ft}$. The altitudes of the bottoms of the total potential scour holes at the right abutment, referenced to the low chord of the bridge at $497.5 \mathrm{ft}$, are $489,488,488$, and $481 \mathrm{ft}$. The abutment scour depth profile is not shown in figure 2 because the values for abutment scour are not considered to be reliable. 


\section{REFERENCES CITED}

Arcement, G.J. and Schneider, V.R., 1984, Guide for selecting Manning's roughness coefficients for natural channels and flood plains, Federal Highway Administration Report FHWA-TS-84-204, 62 p.

Arneson, L.A., Shearman, J.O., Jones, J.S., 1992, Evaluating scour at bridges using WSPRO: Transportation Research Board Draft Paper, 40 p.

Federal Emergency Management Agency, 1992, Flood Insurance Study, Town of Amesbury, Massachusetts, Middlesex County, $20 \mathrm{p}$.

Folk, R.L., 1980, Petrology of sedimentary rocks: Hemphill Publishing Company, Austin, Texas, $184 \mathrm{p}$.

Hayes, D.C., 1993, Site selection and collection of bridgescour data in Delaware, Maryland, and Virginia: U.S. Geological Survey Water-Resources Investigations Report 93-4017, 23 p.
Richardson, E.V., and Davis, S.R., 1995, Evaluating scour at bridges: Federal Highway Administration Hydraulic Engineering Circular No. 18, (HEC-18), Publication FHWA-IP-90-017, 204 p.

Shearman, J.O., Kirby, W.H., Schneider, V.R., Flippo, H.N., 1986, Bridge waterways analysis model: research report, Federal Highway Administration Report FHWA/RD-86/108, $126 \mathrm{p}$.

Shearman, J.O., 1990, User's manual for WSPRO--A computer model for water surface profile computations, FHWA-IP-89-027, September, 1990.

Wandle, S.W., Jr., 1983, Estimating peak discharges of small, rural streams in Massachusetts, U.S. Geological Survey Water-Supply Paper 2214, 26 p. 


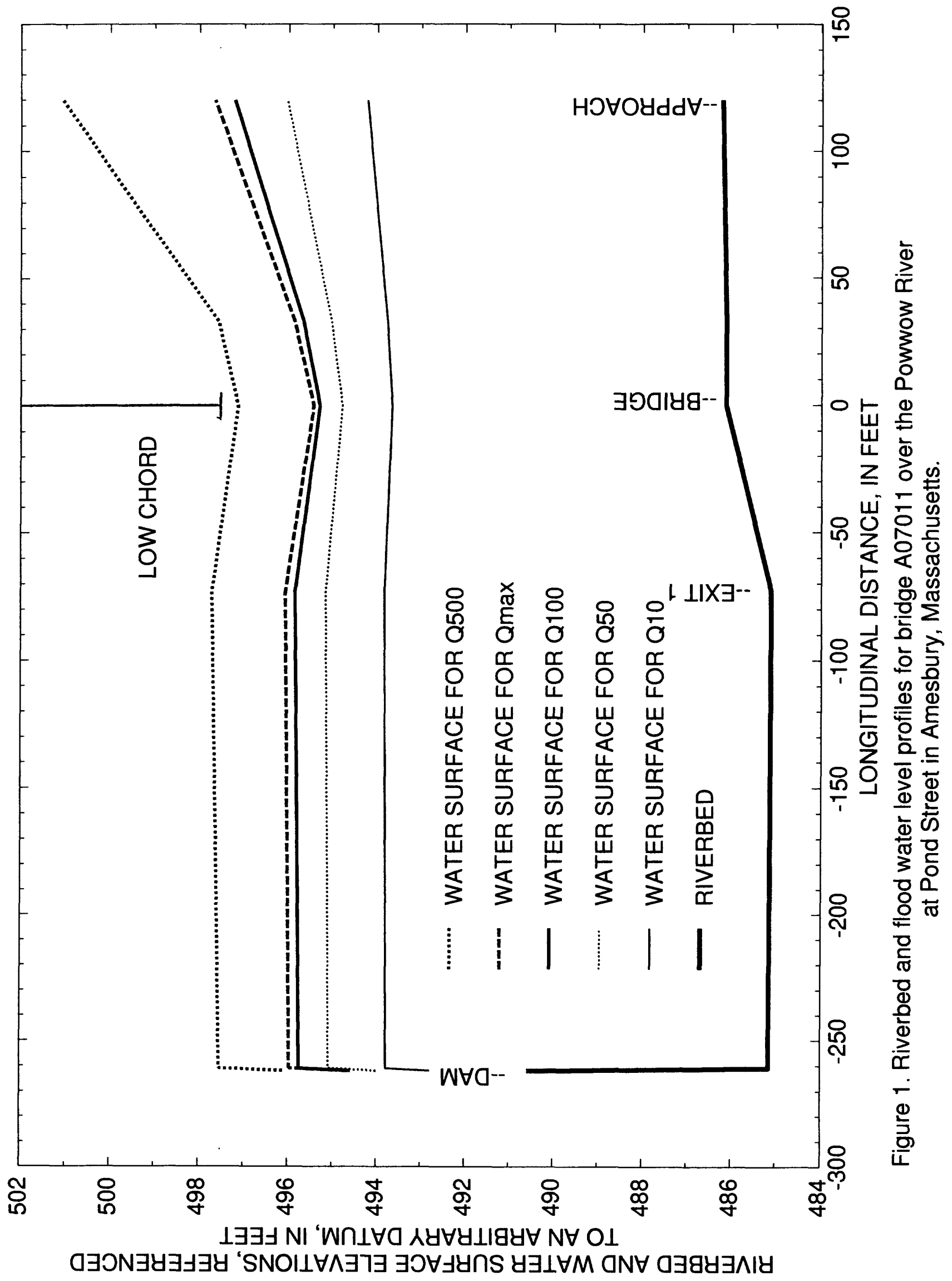




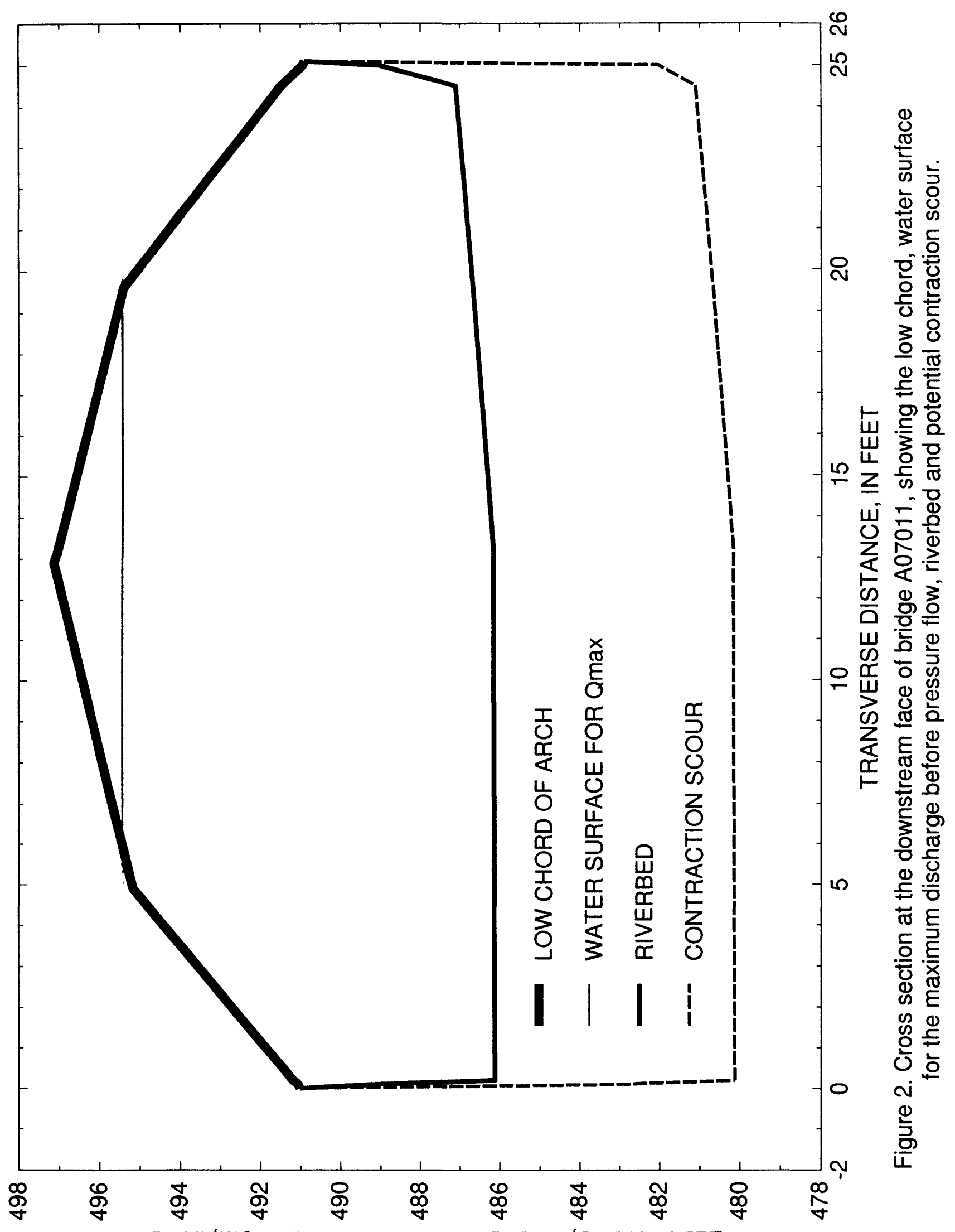

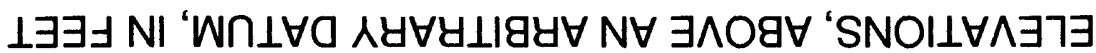

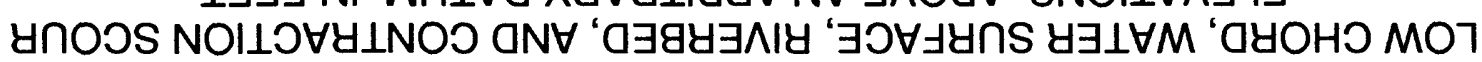


TABLES 1-5

- PAGE II FOLLOWS- 
Table 1. Summary of potential scour depths for bridge A07011, over the Powwow River at Pond Street in Amesbury, Massachusetts

[ft, foot; $\mathrm{ft}^{3} / \mathrm{s}$, cubic foot per second]

\begin{tabular}{|c|c|c|c|c|c|c|}
\hline \multirow{2}{*}{$\begin{array}{l}\text { Flood return } \\
\text { period }\end{array}$} & \multirow{2}{*}{$\begin{array}{c}\text { Discharge, } \\
\mathrm{ft}^{3} / \mathrm{s}\end{array}$} & \multirow{2}{*}{$\begin{array}{l}\text { Bed transport } \\
\text { condition }\end{array}$} & \multicolumn{4}{|c|}{ Scour depths (ft) } \\
\hline & & & Contraction & Left abutment & Right abutment & Pier \\
\hline 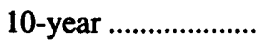 & 921 & clear water & 0 & 7 & 4 & None \\
\hline 50-year ................... & 1,550 & clear water & 3 & 10 & 6 & None \\
\hline 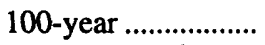 & 1,990 & clear water & 5 & 11 & 7 & None \\
\hline Pressure flow ${ }^{1}$......... & 2,150 & clear water & 6 & 12 & 7 & None \\
\hline
\end{tabular}

${ }^{1}$ Maximum discharge that can pass under the bridge before reaching pressure flow and road overtopping. The flood return period is undetermined.

Table 2. Determination of live-bed or clear-water conditions for bridge A07011, over the Powwow River at Pond Street in Amesbury, Massachusetts

[Symbol: $a$, approach section; $m$, main channel subarea; $C$, critical; $M$, mean; $t$, total. Pressure flow: Maximum discharge that can pass under the bridge before reaching pressure flow and road overtopping. The flood return period is undetermined. $\mathrm{ft}$, foot; $\mathrm{ft}^{2}$, square foot; $\mathrm{f} / \mathrm{s}$, foot per second; $\mathrm{ft}^{3} / \mathrm{s}$, cubic foot per second]

\begin{tabular}{|c|c|c|c|c|c|}
\hline \multirow{2}{*}{ Parameter } & \multirow{2}{*}{ Symbol } & \multicolumn{4}{|c|}{ Value for Indicated recurrence interval } \\
\hline & & 10-year & 50-year & 100-year & Pressure flow \\
\hline \multicolumn{6}{|l|}{ Approach Section } \\
\hline Total discharge, $\mathrm{ft}^{3} / \mathrm{s}$ & $Q_{\mathrm{t}}(a)$ & 921 & 1,550 & 1,990 & 2,150 \\
\hline Total conveyance, $\mathrm{ft}^{3} / \mathrm{s}$ & $K_{\mathrm{t}}(a)$ & 47,700 & 71,500 & 89,300 & 96,300 \\
\hline Water-surface elevation above arbitrary datum, $\mathrm{ft}$ & $h(a)$ & 494.23 & 496.04 & 497.22 & 497.66 \\
\hline Conveyance of main channel, $\mathrm{ft}^{3} / \mathrm{s}$ & $K\left(a_{m}\right)$ & 43,900 & 63,800 & 78,300 & 84,000 \\
\hline Area of main channel, $\mathrm{ft}^{2}$ & $A\left(a_{m}\right)$ & 281 & 351 & 397 & 414 \\
\hline Top width of main channel, $\mathrm{ft}$ & $T\left(a_{m}\right)$ & 39 & 39 & 39 & 39 \\
\hline Median grain size, $\mathrm{ft}$ & $d_{50}\left(a_{m}\right)$ & 0.035 & 0.035 & 0.035 & 0.035 \\
\hline \multicolumn{6}{|l|}{ Calculated Parameters } \\
\hline Discharge, $\mathrm{ft}^{3} / \mathrm{s},\left[K\left(a_{m}\right) / K_{\mathrm{t}}(a)\right] Q_{\mathrm{t}}(a)$ & $Q\left(a_{m}\right)$ & 848 & 1,380 & 1,740 & 1,880 \\
\hline Mean water depth, ft, $A\left(a_{m}\right) / T\left(a_{m}\right)$ & $y\left(a_{m}\right)$ & 7.2 & 9.0 & 10.2 & 10.6 \\
\hline Mean velocity, $\mathrm{ft} / \mathrm{s}, Q\left(a_{m}\right) / A\left(a_{m}\right)$ & $V_{\mathbf{M}}\left(a_{m}\right)$ & 3.0 & 3.9 & 4.4 & 4.5 \\
\hline Critical velocity, $\mathrm{ft} / \mathrm{s}$, Neill equation ${ }^{1}$ & $V_{\mathrm{C}}\left(a_{m}\right)$ & 5.1 & 5.3 & 5.4 & 5.4 \\
\hline \multicolumn{6}{|l|}{ Results } \\
\hline $\begin{array}{l}\text { Live bed }\left[V_{M}\left(a_{m}\right)>V_{C}\left(a_{m}\right)\right] \text { or Clear water } \\
{\left[V_{M}\left(a_{m}\right)<V_{C}\left(a_{m}\right)\right]}\end{array}$ & & Clear water & Clear water & Clear water & Clear water \\
\hline
\end{tabular}


Table 3. Calculation of clear-water contraction scour for bridge A07011, over the Powwow River at Pond Street in Amesbury, Massachusetts

[Symbol: $a$, approach section; $c$, downstream bridge-face section; $m$, main channel subarea; $\mathrm{p}$, pier; $\mathrm{t}$, total. Pressure flow: Maximum discharge that can pass under the bridge before reaching pressure flow and road overtopping. The flood return period is undetermined. $\mathrm{ft}$, foot; $\mathrm{ft}^{2}$, square foot; $\mathrm{ft} / \mathrm{s}$, foot per second; $\mathrm{ft}^{3} / \mathrm{s}$, cubic foot per second]

\begin{tabular}{|c|c|c|c|c|c|}
\hline \multirow{2}{*}{ Parameter } & \multirow{2}{*}{ Symbol } & \multicolumn{4}{|c|}{ Value for indicated recurrence interval } \\
\hline & & 10-year & 50-year & 100-year & Pressure flow \\
\hline \multicolumn{6}{|l|}{ Approach Section } \\
\hline Total discharge, $\mathrm{ft}^{3} / \mathrm{s}$ & $Q_{\mathrm{t}}(a)$ & 921 & 1,550 & 1,990 & 2,150 \\
\hline Water-surface elevation above arbitrary datum, $\mathrm{ft}$ & $h(a)$ & 494.23 & 496.04 & 497.22 & 497.66 \\
\hline Area of main channel, $\mathrm{ft}^{2}$ & $A\left(a_{m}\right)$ & 281 & 351 & 397 & 414 \\
\hline Top width of main channel, $\mathrm{ft}$ & $T\left(a_{m}\right)$ & 39 & 39 & 39 & 39 \\
\hline \multicolumn{6}{|l|}{ Downstream Bridge-Face Section } \\
\hline Total discharge through bridge, $\mathrm{ft}^{3} / \mathrm{s}$ & $Q_{t}(c)$ & 921 & 1,550 & 1,990 & 2,150 \\
\hline Total conveyance through bridge, $\mathrm{ft}^{3} / \mathrm{s}$ & $K_{\mathrm{t}}(c)$ & 18,200 & 20,600 & 21,400 & 21,500 \\
\hline Water-surface elevation above arbitrary datum, $\mathrm{ft}$ & $h(c)$ & 493.67 & 494.79 & 495.30 & 495.44 \\
\hline Conveyance of main channel, $\mathrm{ft}^{3} / \mathrm{s}$ & $K\left(c_{m}\right)$ & 18,200 & 20,600 & 21,400 & 21,500 \\
\hline Area of main channel, $\mathrm{ft}^{2}$ & $A\left(c_{m}\right)$ & 167 & 185 & 193 & 195 \\
\hline Total bottom width of main channel (including piers), $\mathrm{ft}$ & $B_{t}\left(c_{m}\right)$ & 25 & 25 & 25 & 25 \\
\hline $\begin{array}{l}\text { Total width of piers in main channel, } \mathrm{ft} \text { (width } \\
\text { measured at base of pier) }\end{array}$ & $W_{\mathrm{p}}\left(c_{m}\right)$ & $\mathbf{0}$ & 0 & 0 & 0 \\
\hline Median grain size, $\mathrm{ft}$ & $d_{50}\left(c_{m}\right)$ & 0.035 & 0.035 & 0.035 & 0.035 \\
\hline \multicolumn{6}{|l|}{ Calculated Parameters } \\
\hline Discharge, $\mathrm{ft}^{3} / \mathrm{s},\left[K\left(c_{m}\right) / K_{\mathrm{t}}(c)\right] Q_{\mathrm{t}}(c)$ & $Q\left(c_{m}\right)$ & 921 & 1,550 & 1,990 & 2,150 \\
\hline $\begin{array}{l}\text { Diameter of smallest non-transportable bed material, } \mathrm{ft} \text {, } \\
1.25 d_{50}\left(c_{m}\right)\end{array}$ & $d_{\mathrm{n}}\left(c_{m}\right)$ & 0.044 & 0.044 & 0.044 & 0.044 \\
\hline $\begin{array}{l}\text { Adjusted bottom width of main channel, } \mathrm{ft} \\
B_{\mathrm{t}}\left(c_{m}\right)-W_{\mathrm{p}}\left(c_{m}\right)\end{array}$ & $B\left(c_{m}\right)$ & 25 & 25 & 25 & 25 \\
\hline Mean water depth at approach, ft, $A\left(a_{m}\right) / T\left(a_{m}\right)$ & $y\left(a_{m}\right)$ & 7.21 & 9.00 & 10.2 & 10.6 \\
\hline $\begin{array}{l}\text { Mean water depth at downstream bridge face, } \mathrm{ft} \\
\qquad A\left(c_{m}\right) / B\left(c_{m}\right)\end{array}$ & $y\left(c_{m}\right)$ & 6.68 & 7.40 & 7.72 & 7.80 \\
\hline $\begin{array}{l}\text { Mean water depth including contraction scour, } \mathrm{ft} \text {, } \\
\text { Laursen's } 1963 \text { equation }{ }^{1}\end{array}$ & $y_{2}\left(c_{m}\right)$ & 6.66 & 10.4 & 12.9 & 13.8 \\
\hline \multicolumn{6}{|l|}{ Results } \\
\hline $\begin{array}{l}\text { Difference in mean water depth between approach and } \\
\text { bridge sections, ft, } y_{2}\left(c_{m}\right)-y\left(a_{m}\right)\end{array}$ & $y_{\mathrm{d}}\left(c_{m}\right)$ & 0 & 1 & 3 & 3 \\
\hline Mean scour depth at bridge, ft, $y_{2}\left(c_{m}\right)-y\left(c_{m}\right)$ & $y_{s}\left(c_{m}\right)$ & 0 & 3 & 5 & 6 \\
\hline
\end{tabular}


Table 4. Calculation of local scour at the left abutment for bridge A07011, over the Powwow River at Pond Street in Amesbury, Massachusetts

[ Symbol: $a$, approach section; $b$, upstream bridge face section; $R$, roadway section; $l$, left overbank subarea; E, embankment; F, flowtube; P, projected; t, total. Pressure flow: Maximum discharge that can pass under the bridge before reaching pressure flow and road overtopping. The flood return period is undetermined.; $\mathrm{ft}$, foot; $\mathrm{ft}^{2}$, square foot; $\mathrm{ft} / \mathrm{s}$, foot per second; $\mathrm{ft}^{3} / \mathrm{s}$, cubic foot per second]

\begin{tabular}{|c|c|c|c|c|c|}
\hline \multirow{2}{*}{ Parameter } & \multirow{2}{*}{ Symbol } & \multicolumn{4}{|c|}{ Value for indicated recurrence interval } \\
\hline & & 10-year & 50-year & 100-year & Pressure flow \\
\hline \multicolumn{6}{|l|}{ Approach and Roadway Sections } \\
\hline Total discharge, $\mathrm{ft}^{3} / \mathrm{s}$ & $Q_{\mathrm{t}}(a)$ & 921 & 1,550 & 1,990 & 2,150 \\
\hline $\begin{array}{l}\text { Area of approach section determined by projection of the left } \\
\text { embankment, } \mathrm{ft}^{2}\end{array}$ & $A_{\mathrm{P}}\left(a_{l}\right)$ & 60 & 97 & 124 & 135 \\
\hline Length of left embankment projected onto approach section, $\mathrm{ft}$ & $T_{\mathrm{P}}\left(a_{l}\right)$ & 19 & 22 & 24 & 25 \\
\hline Total conveyance, $\mathrm{ft}^{3} / \mathrm{s}$ & $K_{\mathrm{t}}(a)$ & 47,700 & 71,500 & 89,300 & 96,300 \\
\hline $\begin{array}{l}\text { Conveyance obstructed by projecting left embankment onto } \\
\text { approach section, } \mathrm{ft}^{3 / s}\end{array}$ & $K_{\mathrm{P}}\left(a_{l}\right)$ & 3,130 & 6,230 & 8,810 & 9,880 \\
\hline \multicolumn{6}{|l|}{ Parameters and Calculations for Road Overflow } \\
\hline Discharge over left roadway, $\mathrm{ft}^{3} / \mathrm{s}$ & $Q\left(R_{l}\right)$ & 0 & 0 & 0 & 0 \\
\hline Discharge per equal conveyance flowtube, $\mathrm{ft}^{3} / \mathrm{s}, Q_{\mathrm{t}}(a) / 20$ & $q_{\mathrm{F}}$ & 46.0 & 77.6 & 99.4 & 108 \\
\hline $\begin{array}{l}\text { Number of conveyance tubes corresponding to road overflow } \\
\text { discharge, } Q\left(R_{l}\right) / q_{\mathrm{F}}\end{array}$ & \# tubes & 0 & 0 & 0 & 0 \\
\hline $\begin{array}{l}\text { Width of conveyance tube corresponding to road overflow } \\
\text { discharge, ft. (From HP-2 output). }\end{array}$ & $L\left(R_{l}\right)$ & 0 & 0 & 0 & 0 \\
\hline Area of conveyance tube corresponding to road overflow, $\mathrm{ft}^{2}$ & $A\left(R_{l}\right)$ & 0 & 0 & 0 & 0 \\
\hline \multicolumn{6}{|l|}{ Calculated Parameters } \\
\hline Embankment length blocking flow, ft, $T_{\mathrm{P}}\left(a_{l}\right)-L\left(R_{l}\right)$ & $L_{\mathrm{E}}\left(a_{l}\right)$ & 19 & 22 & 24 & 25 \\
\hline Area of flow blocked by embankment, $\mathrm{ft}^{2}, A_{\mathrm{p}}\left(a_{l}\right)-A\left(R_{l}\right)$ & $\mathrm{A}_{\mathrm{E}}\left(a_{l}\right)$ & 60 & 97 & 124 & 135 \\
\hline $\begin{array}{l}\text { Average depth of flow blocked by embankment, ft, } \\
\qquad A_{\mathrm{E}}\left(a_{l}\right) / L_{\mathrm{E}}\left(a_{l}\right)\end{array}$ & $Y_{\mathrm{E}}(a l)$ & 3.16 & 4.41 & 5.17 & 5.40 \\
\hline $\begin{array}{l}\text { Discharge determined by projection of embankment onto } \\
\text { approach section, } \mathrm{ft}^{3} / \mathrm{s},\left[K_{\mathrm{P}}\left(a_{l}\right) / K_{\mathrm{t}}(a)\right] Q_{\mathrm{t}}(a)\end{array}$ & $Q_{\mathrm{P}}\left(a_{l}\right)$ & 60.5 & 135 & 196 & 221 \\
\hline Discharge blocked by embankment, $\mathrm{ft}^{3} / \mathrm{s}, Q_{\mathrm{P}}\left(a_{l}\right)-Q\left(R_{l}\right)$ & $Q_{\mathrm{E}}\left(a_{l}\right)$ & 60.5 & 135 & 196 & 221 \\
\hline $\begin{array}{l}\text { Average velocity of flow blocked by embankment, ft/s, } \\
\qquad Q_{\mathrm{E}}\left(a_{l}\right) / A_{\mathrm{E}}\left(a_{l}\right)\end{array}$ & $V_{\mathrm{E}}\left(a_{l}\right)$ & 1.01 & 1.39 & 1.58 & 1.64 \\
\hline $\begin{array}{l}\text { Froude number of flow blocked by embankment, } \\
\qquad V_{\mathrm{E}}\left(a_{l}\right) /\left[32.2 Y_{\mathrm{E}}\left(a_{l}\right)\right]^{0.5}\end{array}$ & $F r_{\mathrm{E}}\left(a_{l}\right)$ & 0.10 & 0.12 & 0.12 & 0.12 \\
\hline $\begin{array}{l}\text { Correction factor for abutment type: } \\
1.00, \text { for vertical abutment; or } \\
0.82 \text {, for vertical abutment with wingwall; or } 0.55 \text {, } \\
\text { for spillthrough abutment }\end{array}$ & $k_{1}\left(b_{l}\right)$ & 1.00 & 1.00 & 1.00 & 1.00 \\
\hline $\begin{array}{l}\text { Angle of embankment to flow, degrees: } \\
\begin{aligned} \theta & =90 \text { if embankment is normal to flow, or } \\
\theta & <90 \text { if embankment is angled downstream, or } \\
\theta & >90 \text { if embankment is angled upstream }\end{aligned}\end{array}$ & $\boldsymbol{\theta}$ & 90 & 90 & 90 & 90 \\
\hline Correction factor for angle of embankment to flow, $(\theta / 90)^{0.13}$ & $k_{2}\left(b_{l}\right)$ & 1.00 & 1.00 & 1.00 & 1.00 \\
\hline \multicolumn{6}{|l|}{ Results } \\
\hline Scour depth, $\mathrm{ft}$, Froehlich equation ${ }^{1}$ & $y_{\mathrm{s}}\left(b_{l}\right)$ & 7 & 10 & 11 & 12 \\
\hline
\end{tabular}

${ }^{\mathrm{T}}$ Froehlich equation, $y_{\mathrm{s}}(\mathrm{b} l)=\left(2.27 k_{1}\left(b_{l}\right) k_{2}\left(b_{l}\right)\left[L_{\mathrm{E}}\left(a_{l}\right) / Y_{\mathrm{E}}(a l)\right]^{0.43}\left[F r_{\mathrm{E}}\left(a_{l}\right)\right]^{0.61}+1\right\} Y_{\mathrm{E}}\left(a_{l}\right)$ (Richardson and Davis, 1995, p. 48, eqn. 28). 
Table 5. Calculation of local scour at the right abutment for bridge A07011, over the Powwow River at Pond Street in Amesbury, Massachusetts

[Symbol: $a$, approach section; $b$, upstream bridge-face section; $R$, roadway section; $r$, right overbank subarea; $\mathrm{E}$, embankment; $\mathrm{F}$, flowtube; $\mathrm{P}$, projected; $t$, total; Pressure flow: Maximum discharge that can pass under the bridge before reaching pressure flow and road overtopping. The flood retum period is undetermined. $\mathrm{ft}$, foot; $\mathrm{ft}^{2}$, square foot; $\mathrm{ft} / \mathrm{s}$, foot per second; $\mathrm{ft}^{3} / \mathrm{s}$, cubic foot per second]

\begin{tabular}{|c|c|c|c|c|c|}
\hline \multirow{2}{*}{ Parameter } & \multirow{2}{*}{ Symbol } & \multicolumn{4}{|c|}{ Value for Indlcated recurrence Interval } \\
\hline & & 10-year & 50-year & 100-year & Pressure flow \\
\hline \multicolumn{6}{|l|}{ Approach and Roadway Sections } \\
\hline Total discharge, $\mathrm{ft}^{3} / \mathrm{s}$ & $Q_{t}(a)$ & 921 & 1,550 & 1,990 & 2,150 \\
\hline $\begin{array}{l}\text { Area of approach section determined by projection of the right } \\
\text { abutment, } \mathrm{ft}^{2}\end{array}$ & $A_{\mathrm{P}}\left(a_{r}\right)$ & 17 & 31 & 43 & 49 \\
\hline Length of right embankment projected onto approach section, $\mathrm{ft}$ & $T_{\mathrm{P}}\left(a_{r}\right)$ & 7 & 9 & 13 & 16 \\
\hline Total conveyance, $\mathrm{ft}^{3} / \mathrm{s}$ & $K_{\mathrm{t}}(a)$ & 47,700 & 71,500 & 89,300 & 96,300 \\
\hline $\begin{array}{l}\text { Conveyance obstructed by projecting right embankment onto } \\
\text { approach section, } \mathrm{ft}^{\mathrm{t}} / \mathrm{s}\end{array}$ & $K_{\mathrm{P}}\left(a_{r}\right)$ & 3,130 & 6,230 & 8,810 & 9,880 \\
\hline \multicolumn{6}{|l|}{ Parameters and Calculations for Road Overflow } \\
\hline Discharge over right roadway, $\mathrm{ft}^{3} / \mathrm{s}$ & $Q\left(R_{r}\right)$ & 0 & 0 & 0 & 0 \\
\hline Discharge per equal conveyance flowtube, $\mathrm{ft}^{3} / \mathrm{s}, Q_{\mathrm{t}}(a) / 20$ & $q_{\mathrm{F}}$ & 46.0 & 77.6 & 99.4 & 108 \\
\hline $\begin{array}{l}\text { Number of conveyance tubes corresponding to road overflow } \\
\text { discharge, } Q\left(R_{r}\right) / q_{\mathrm{F}}\end{array}$ & \# tubes & 0 & 0 & 0 & 0 \\
\hline $\begin{array}{l}\text { Width of conveyance tube corresponding to road overflow } \\
\text { discharge, ft. (From HP-2 output). }\end{array}$ & $L\left(R_{r}\right)$ & 0 & 0 & 0 & 0 \\
\hline $\begin{array}{l}\text { Area of conveyance tube corresponding to road overflow } \\
\text { discharge, } \mathrm{ft}^{2} \text {. (From HP-2 output). }\end{array}$ & $A\left(R_{r}\right)$ & 0 & 0 & 0 & 0 \\
\hline \multicolumn{6}{|l|}{ Calculated Parameters } \\
\hline Embankment length blocking flow, $\mathrm{ft}, T_{\mathrm{P}}\left(a_{r}\right)-L\left(R_{r}\right)$ & $L_{\mathrm{E}}\left(a_{r}\right)$ & 7 & 9 & 13 & 16 \\
\hline Area of flow blocked by embankment, $\mathrm{ft}^{2}, A_{\mathrm{P}}\left(a_{r}\right)-A\left(R_{r}\right)$ & $A_{\mathrm{E}}\left(a_{r}\right)$ & 17 & 31 & 43 & 49 \\
\hline $\begin{array}{l}\text { Average depth of flow blocked by embankment, } \mathrm{ft} \\
\qquad A_{\mathrm{E}}\left(a_{r}\right) / L_{\mathrm{E}}\left(a_{r}\right)\end{array}$ & $Y_{\mathrm{E}}\left(a_{r}\right)$ & 2.43 & 3.44 & 3.31 & 3.06 \\
\hline $\begin{array}{l}\text { Discharge determined by projection of embankment onto } \\
\text { approach section, } \mathrm{ft}^{3} / \mathrm{s},\left[K_{\mathrm{P}}\left(a_{r}\right) / K_{\mathrm{t}}(a)\right] Q_{\mathrm{t}}(a)\end{array}$ & $Q_{\mathrm{P}}\left(a_{r}\right)$ & 12.7 & 32.5 & 47.3 & 52.2 \\
\hline Discharge blocked by embankment, $\mathrm{ft}^{3} / \mathrm{s}, Q_{\mathrm{P}}\left(a_{r}\right) \cdot Q\left(R_{r}\right)$ & $Q_{\mathrm{E}}\left(a_{r}\right)$ & 12.7 & 32.5 & 47.3 & 52.2 \\
\hline $\begin{array}{l}\text { Average velocity of flow blocked by embankment, } \mathrm{ft} / \mathrm{s} \text {, } \\
\qquad Q_{\mathrm{E}}\left(a_{r}\right) / A_{\mathrm{E}}\left(a_{r}\right)\end{array}$ & $V_{\mathrm{E}}\left(a_{r}\right)$ & 0.75 & 1.05 & 1.10 & 1.07 \\
\hline $\begin{array}{l}\text { Froude number of flow blocked by embankment, } \\
\qquad V_{\mathrm{E}}\left(a_{r}\right) /\left[32.2 Y_{\mathrm{E}}\left(a_{r}\right)\right]^{0.5}\end{array}$ & $F r_{\mathrm{E}}\left(a_{r}\right)$ & 0.08 & 0.10 & 0.11 & 0.11 \\
\hline $\begin{array}{l}\text { Correction factor for abutment type:, } \\
1.0 \text {, for vertical abutment; or } \\
0.82 \text {, for vertical abutment with wingwall; or } 0.55 \text {, for } \\
\text { spill-through abutment }\end{array}$ & $k_{1}\left(b_{r}\right)$ & 1.00 & 1.00 & 1.00 & 1.00 \\
\hline $\begin{array}{l}\text { Angle of embankment to flow, degrees: } \\
\begin{array}{l}\theta=90 \text { if embankment is normal to flow, or } \\
\theta<90 \text { if embankment is angled downstream, or } \\
\theta>90 \text { if embankment is angled upstream }\end{array}\end{array}$ & $\boldsymbol{\theta}$ & 90 & 90 & 90 & 90 \\
\hline Correction factor for angle of embankment to flow, $(\theta / 90)^{0.13}$ & $k_{2}\left(b_{r}\right)$ & 1.00 & 1.00 & 1.00 & 1.00 \\
\hline \multicolumn{6}{|l|}{ Results } \\
\hline Scour depth, ft, Froehlich equation ${ }^{1}$ & $y_{s}\left(b_{r}\right)$ & 4 & 6 & 7 & 7 \\
\hline
\end{tabular}

${ }^{T}$ Froehlich equation, $y_{s}\left(b_{r}\right)=\left\{2.27 k_{1}\left(b_{r}\right) k_{2}\left(b_{r}\right)\left[L_{\mathrm{E}}\left(a_{r}\right) / Y_{\mathrm{E}}\left(a_{r}\right)\right]^{0.43}\left[F r_{\mathrm{E}}\left(a_{r}\right)\right]^{0.61}+1\right\} Y_{\mathrm{E}}\left(a_{r}\right)$ (Richardson and Davis, 1995, p. 48, eqn. 28) 


\author{
APPENDIX A \\ WSPRO Input Data File for \\ Bridge A07011, Amesbury, Massachusetts
}


GR

GR

U.S. Geological Survey WSPRO Input File a07011.wsp Hydraulic analysis for structure a07011 a07011 awispp.x a07011.dca.7 downstream 1a $\begin{array}{llllll}921 & 1553 & 1989 & 2150 & 2160 & 3628\end{array}$ $\begin{array}{llllll}492.79 & 494.00 & 494.58 & 494.28 & 494.28 & 494.28\end{array}$

$\begin{array}{lllllllllllllllllllll}6 & 29 & 30 & 552 & 553 & 551 & 5 & 16 & 17 & 13 & 3 & * & 15 & 14 & 23 & 21 & 11 & 12 & 4 & 7 & 3\end{array}$

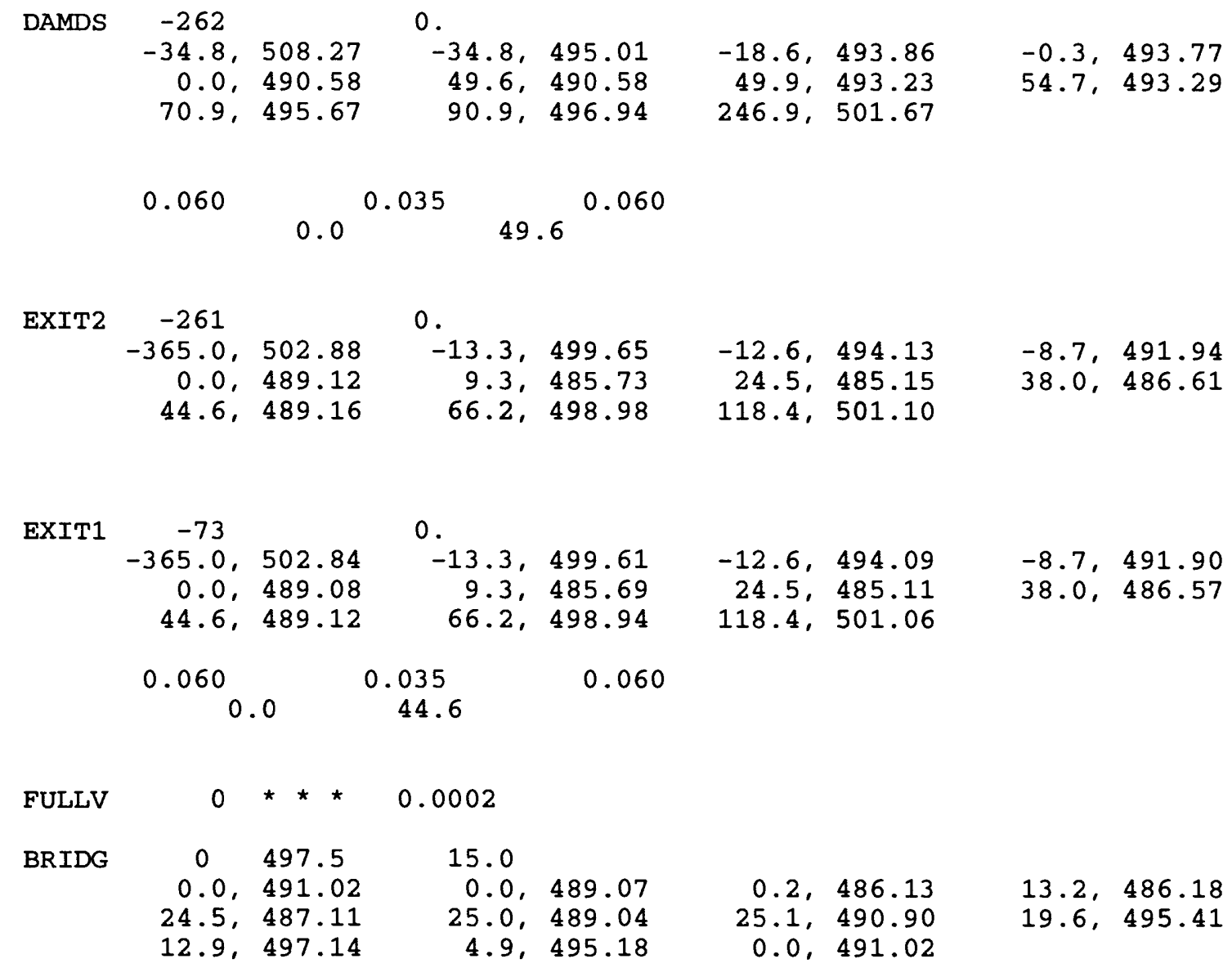


AS

\begin{tabular}{|c|c|}
\hline & \\
\hline & \\
\hline GR & \\
\hline GR & \\
\hline & \\
\hline N & \\
\hline Sz & \\
\hline $\mathrm{PX}$ & DAMDS \\
\hline PX & EXIT1 \\
\hline PX & FULLV \\
\hline PX & BRIDG \\
\hline PX & APPR 1 \\
\hline
\end{tabular}

HP 1 BRIDG

HP 1 BRIDG

HP 1 BRIDG

HP 1 BRIDG

HP 1 BRIDG

493.67

$0.0 \quad 493.67$

494.79

$0.0 \quad 494.79$

495.30

$0.0 \quad 495.30$

$0.0 \quad 495.44$

$0.0 \quad 497.14$

HP 1 APPR 1494.23

$0.0 \quad 494.23$

HP 1 APPR 1496.04

HP 1 APPR 1497.22

HP 1 APPR $1 \quad 497.66$

HP 1 APPR1 500.02

*

EX

ER

$0.0 \quad 496.04$

$0.0 \quad 497.22$

$0.0 \quad 497.66$

$0.0 \quad 500.02$
0 .

$$
\begin{array}{rrr}
23.9,497.24 & -14.7,491.75 \\
18.5,486.22 & 26.8,486.46 \\
49.2,496.89 & 66.6,499.25
\end{array}
$$

$0.0,489.24$

$33.0,486.91$ 


\section{APPENDIX B \\ WSPRO Output File for \\ Bridge A07011, Amesbury, Massachusetts}

- PAGE 21 FOLLOWS- 
WSPRO V082195
FEDERAL HIGHWAY ADMINISTRATION - U. S. GEOLOGICAL SURVEY MODEL FOR WATER-SURFACE PROFILE COMPUTATIONS

* * RUN DATE \& TIME: 09-29-97 14:59

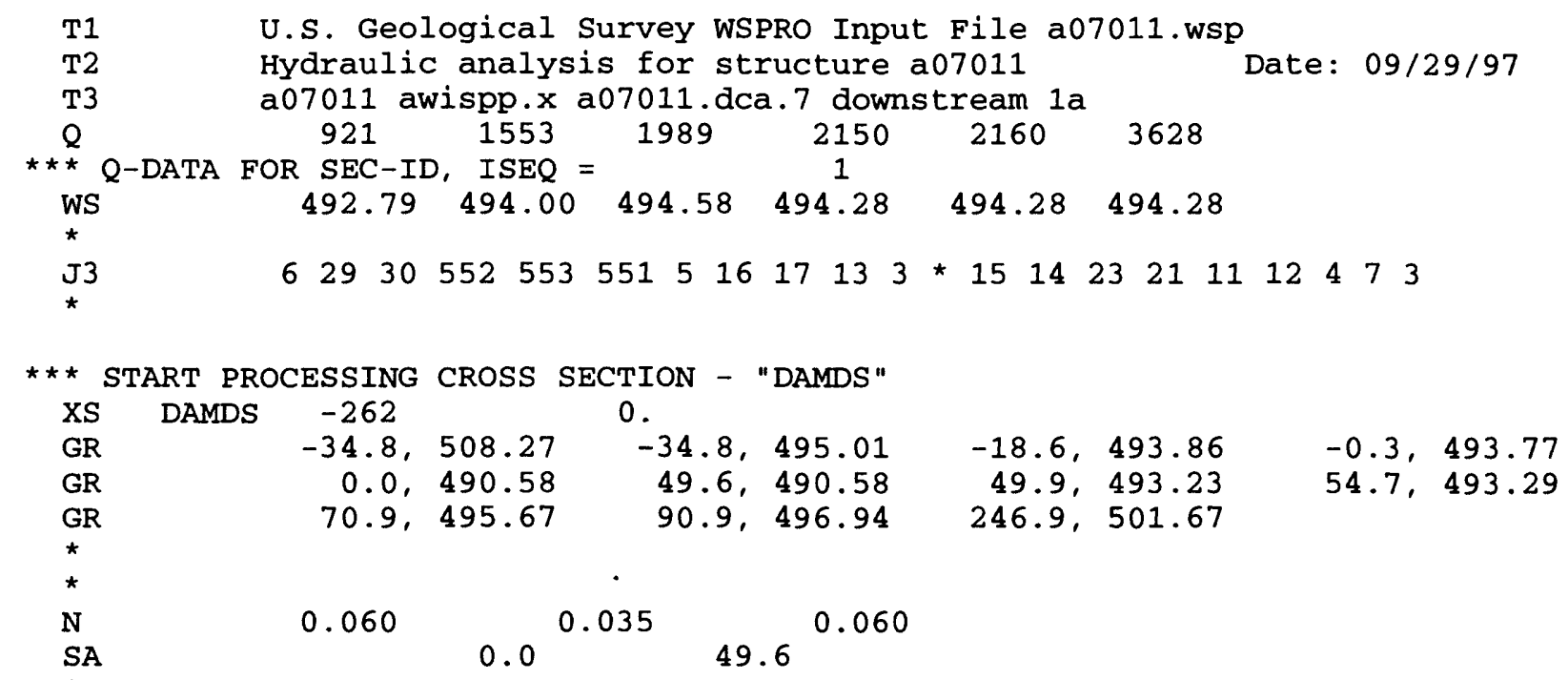

* * FINISH PROCESSING CROSS SECTION - "DAMDS"

** CROSS SECTION "DAMDS" WRITTEN TO DISK, RECORD NO. = 1

-- DATA SUMMARY FOR SECID "DAMDS" AT SRD $=-262$. ERR-CODE =

\begin{tabular}{|c|c|c|c|c|}
\hline EW & IHFNO & VSLOPE & EK & CK \\
\hline
\end{tabular}

\section{$\mathrm{X}-\mathrm{Y}$ COORDINATE PAIRS (NGP $=11):$}

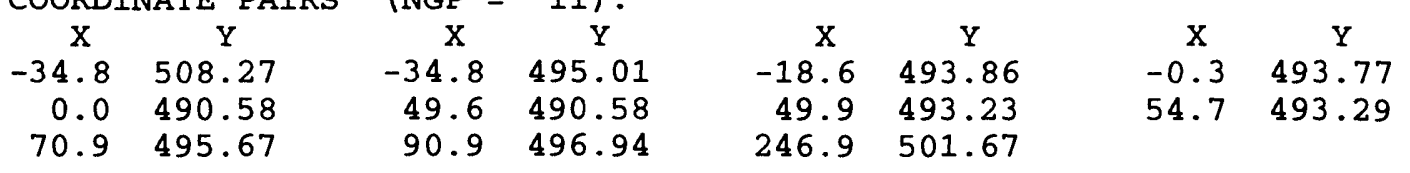

$\mathrm{X}-\mathrm{Y}$ MAX-MIN POINTS:

$\begin{array}{rrrrrrrr}\text { XMIN } & Y & X & \text { YMIN } & \text { XMAX } & Y & X & \text { YMAX } \\ -34.8 & 508.27 & 0.0 & 490.58 & 246.9 & 501.67 & -34.8 & 508.27\end{array}$

SUBAREA BREAKPOINTS (NSA $=3$ ):

$$
0 \text {. } 50 \text {. }
$$

ROUGHNESS COEFFICIENTS (NSA $=3$ ):

1

\footnotetext{
1

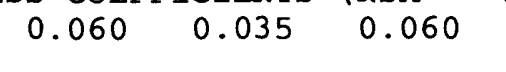

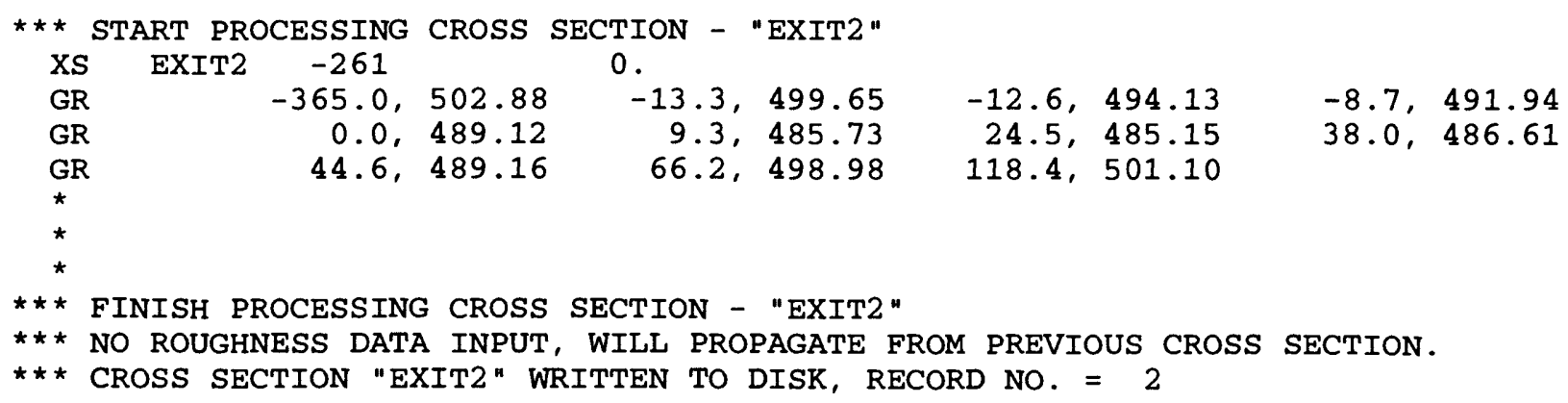




$\begin{array}{rrrrr}\text { SKEW } & \text { IHFNO } & \text { VSLOPE } & \text { EK } & \text { CK } \\ 0.0 & 0 . * \star \star \star \star \star \star \star \star \star & 0.50 & 0.00\end{array}$

$X-Y$ COORDINATE PAIRS (NGP $=11)$ :

$\begin{array}{rrrrrrrr}X & Y & X & Y & X & Y & X & Y \\ -365.0 & 502.88 & -13.3 & 499.65 & -12.6 & 494.13 & -8.7 & 491.94 \\ 0.0 & 489.12 & 9.3 & 485.73 & 24.5 & 485.15 & 38.0 & 486.61 \\ 44.6 & 489.16 & 66.2 & 498.98 & 118.4 & 501.10 & & \end{array}$

$\begin{array}{ccrrrrrr}X-Y & \text { MAX-MIN } & \text { POINTS: } \\ \text { XMIN } & Y & \text { X } & \text { YMIN } & \text { XMAX } & Y & \text { X } & \text { YMAX } \\ -365.0 & 502.88 & 24.5 & 485.15 & 118.4 & 501.10 & -365.0 & 502.88\end{array}$

SUBAREA BREAKPOINTS (NSA $=3$ ):

$$
0 \text {. } 50 \text {. }
$$

ROUGHNESS COEFFICIENTS (NSA = 3):

1

$$
\begin{array}{lll}
0.060 & 0.035 & 0.060
\end{array}
$$$$
\text { * * * START PROCESSING CROSS SECTION - "EXIT1" }
$$$$
\text { XS EXIT1 }-73 \quad 0 \text {. }
$$$$
-365.0,502.84
$$$$
\begin{array}{rr}
-13.3, & 499.61 \\
8 & 9.3,485.69
\end{array}
$$$$
\text { GR } \quad 0.0,489.08
$$$$
\text { GR } \quad 44.6,489.12
$$$$
\begin{array}{rr}
-12.6, & 494.09 \\
24.5, & 485.11 \\
118.4, & 501.06
\end{array}
$$$$
-8.7,491.90
$$

*

N

SA

0.060

0.0

0.035

0.060

$\star$

* * FINISH PROCESSING CROSS SECTION - "EXIT1"

** * CROSS SECTION "EXIT1" WRITTEN TO DISK, RECORD NO. = 3

\begin{tabular}{|c|c|c|c|}
\hline SKEW & IHFNO & $\begin{array}{r}\text { VSLOPE } \\
\star \star \star \star \star \star \star \star \star \star \star \star\end{array}$ & $\begin{array}{rl} & E K \\
0.50 & 50\end{array}$ \\
\hline
\end{tabular}

-- DATA SUMMARY FOR SECID "EXIT1" AT SRD $=-73$. ERR-CODE =

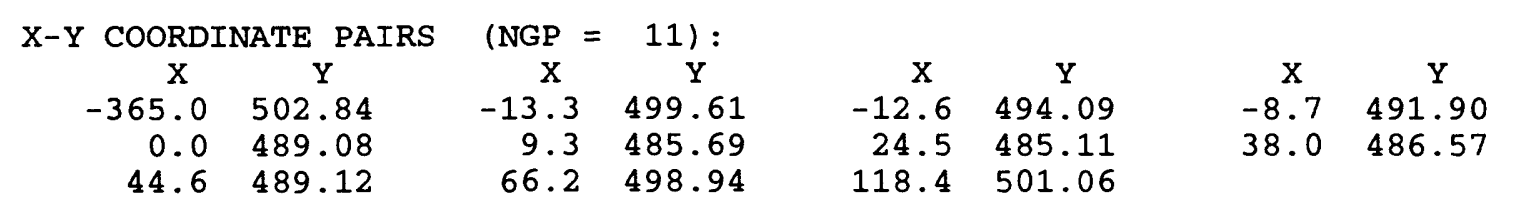

X-Y MAX-MIN POINTS:

$\begin{array}{rrrrrrrr}\text { XMIN } & Y & X & \text { YMIN } & \text { XMAX } & Y & X & \text { YMAX } \\ -365.0 & 502.84 & 24.5 & 485.11 & 118.4 & 501.06 & -365.0 & 502.84\end{array}$

SUBAREA BREAKPOINTS (NSA $=3)$ :

$$
0 \text {. } 45 \text {. }
$$

ROUGHNESS COEFFICIENTS (NSA $=3$ ):

1

$$
\begin{array}{lll}
0.060 & 0.035 & 0.060
\end{array}
$$

$$
\begin{aligned}
& \text { ** * START PROCESSING CROSS SECTION - "FULLV" } \\
& \text { XS FULLV } 0 * \star \star \star 0.0002
\end{aligned}
$$

* * FINISH PROCESSING CROSS SECTION - "FULLV"

$\star \star \star$ NO ROUGHNESS DATA INPUT, WILL PROPAGATE FROM PREVIOUS CROSS SECTION.

$\star \star \star$ CROSS SECTION "FULLV" WRITTEN TO DISK, RECORD NO. = 4 
--- DATA SUMMARY FOR SECID "FULLV" AT SRD =

0. $E R R-C O D E=$ 0

$\begin{array}{rrrrr}\text { SKEW } & \text { IHFNO } & \text { VSLOPE } & \text { EK } & \text { CK } \\ 0.0 & 0 . & 0.0002 & 0.50 & 0.00\end{array}$

$\mathrm{X}-\mathrm{Y}$ COORDINATE PAIRS (NGP $=11)$ :

$\begin{array}{rrrrrrrr}\text { X } & Y & X & Y & X & Y & X & Y \\ -365.0 & 502.85 & -13.3 & 499.62 & -12.6 & 494.10 & -8.7 & 491.91 \\ 0.0 & 489.09 & 9.3 & 485.70 & 24.5 & 485.12 & 38.0 & 486.58 \\ 44.6 & 489.13 & 66.2 & 498.95 & 118.4 & 501.07 & & \end{array}$

$\begin{array}{rrrrrrrr}\text { X-Y MAX-MIN } & \text { POINTS: } \\ \text { XMIN } & Y & \text { X } & \text { YMIN } & \text { XMAX } & Y & \text { X } & \text { YMAX } \\ -365.0 & 502.85 & 24.5 & 485.12 & 118.4 & 501.07 & -365.0 & 502.85\end{array}$

SUBAREA BREAKPOINTS (NSA $=3)$ :

0 . 45 .

ROUGHNESS COEFFICIENTS (NSA = 3 ):

1 $0.060 \quad 0.035 \quad 0.060$

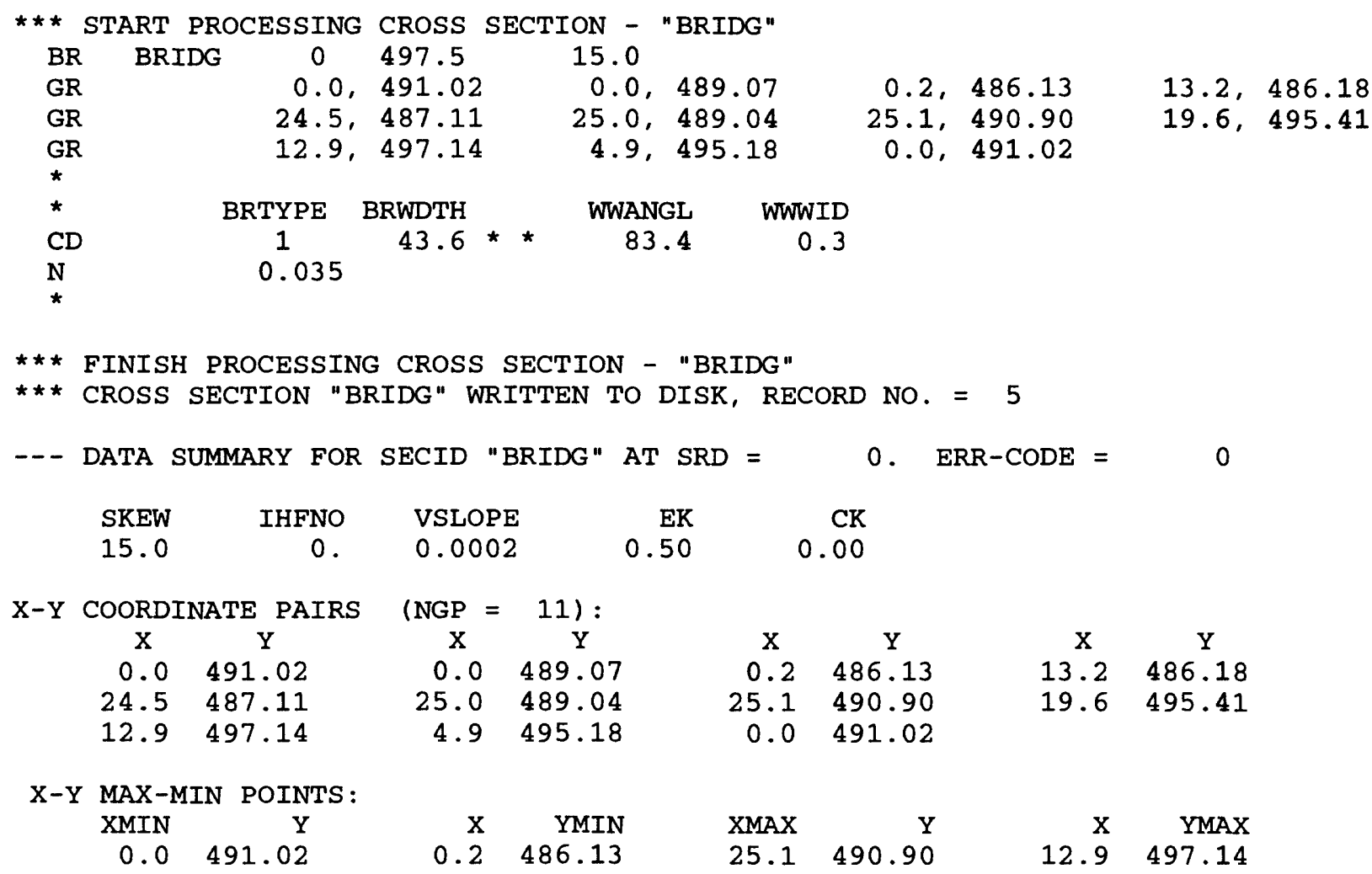

ROUGHNESS COEFFICIENTS (NSA = 1): 0.035

BRIDGE PARAMETERS :

BRTYPE BRWDTH LSEL USERCD WWANGL WWWID ENTRND

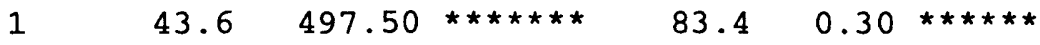
1

PIER DATA: NPW $=0 \quad$ PPCD $=$ *

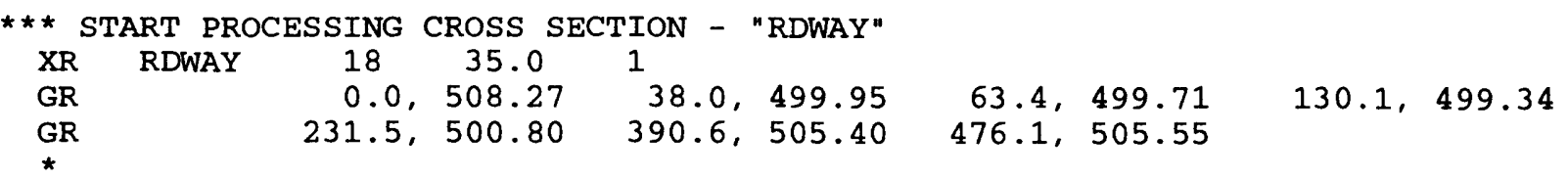




\begin{tabular}{|c|c|c|c|c|c|c|}
\hline * ${ }^{\star} \mathrm{BR}$ & USBRG & $\mathrm{SRD}_{0}$ & $\begin{array}{l}\text { LSEL } \\
\star \star \star \star \star \star \star \star\end{array}$ & $\begin{array}{r}\text { XSSKEW } \\
15.0\end{array}$ & & \\
\hline $\begin{array}{l}\text { * } \mathrm{GR} \\
\text { * } \mathrm{GR} \\
\text { * } \mathrm{GR}\end{array}$ & & $\begin{array}{l}-1.0, \\
11.2, \\
33.5,\end{array}$ & $\begin{array}{l}489.32 \\
485.93 \\
496.99\end{array}$ & $\begin{aligned} 0.0, & 496.99 \\
22.1, & 485.83 \\
-1.0, & 489.32\end{aligned}$ & $\begin{array}{rr}0.0, & 489.11 \\
24.2, & 486.86\end{array}$ & $\begin{array}{rr}2.6, & 487.00 \\
33.5, & 489.11\end{array}$ \\
\hline
\end{tabular}

* WWANGL WWWID

EXPECTED SRD $=35 \mathrm{AT}$ ONE BR. LENGTH BUT COMPUTED SRD $=120$

** * FINISH PROCESSING CROSS SECTION - "RDWAY"

$\star \star \star$ NO ROUGHNESS DATA INPUT, WILL PROPAGATE FROM PREVIOUS CROSS SECTION.

$\star \star \star$ CROSS SECTION "RDWAY" WRITTEN TO DISK, RECORD NO. = 6

--- DATA SUMMARY FOR SECID "RDWAY" AT SRD = 18. $\mathrm{ERR}-\mathrm{CODE}=$ 0
SKEW
IHFNO
VSLOPE
0.0002
$\mathrm{EK}$
0.50
CK

$\mathrm{X}-\mathrm{Y}$ COORDINATE PAIRS (NGP $=7)$ :

$\begin{array}{rrrrrrrr}X & Y & X & Y & X & Y & X & Y \\ 0.0 & 508.27 & 38.0 & 499.95 & 63.4 & 499.71 & 130.1 & 499.34 \\ 231.5 & 500.80 & 390.6 & 505.40 & 476.1 & 505.55 & & \end{array}$
$\mathrm{X}-\mathrm{Y}$ MAX-MIN POINTS :

$\begin{array}{cc}\text { XMIN } & Y \\ 0.0 & 508.27\end{array}$
$\begin{array}{rr}X & \text { YMIN } \\ 130.1 & 499.34\end{array}$
$\begin{array}{rr}\text { XMAX } & Y \\ 476.1 & 505.55\end{array}$
$\begin{array}{rr}X & \text { YMAX } \\ 0.0 & 508.27\end{array}$

SUBAREA BREAKPOINTS (NSA $=3)$ :

$$
0.45 \text {. }
$$

ROUGHNESS COEFFICIENTS
$0.060 \quad 0.035 \quad 0.060$

ROAD GRADE DATA: IPAVE RDWID USERCF

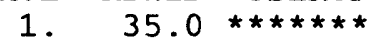

BRIDGE PROJECTION DATA: XREFLT XREFRT FDSTLT FDSTRT

1

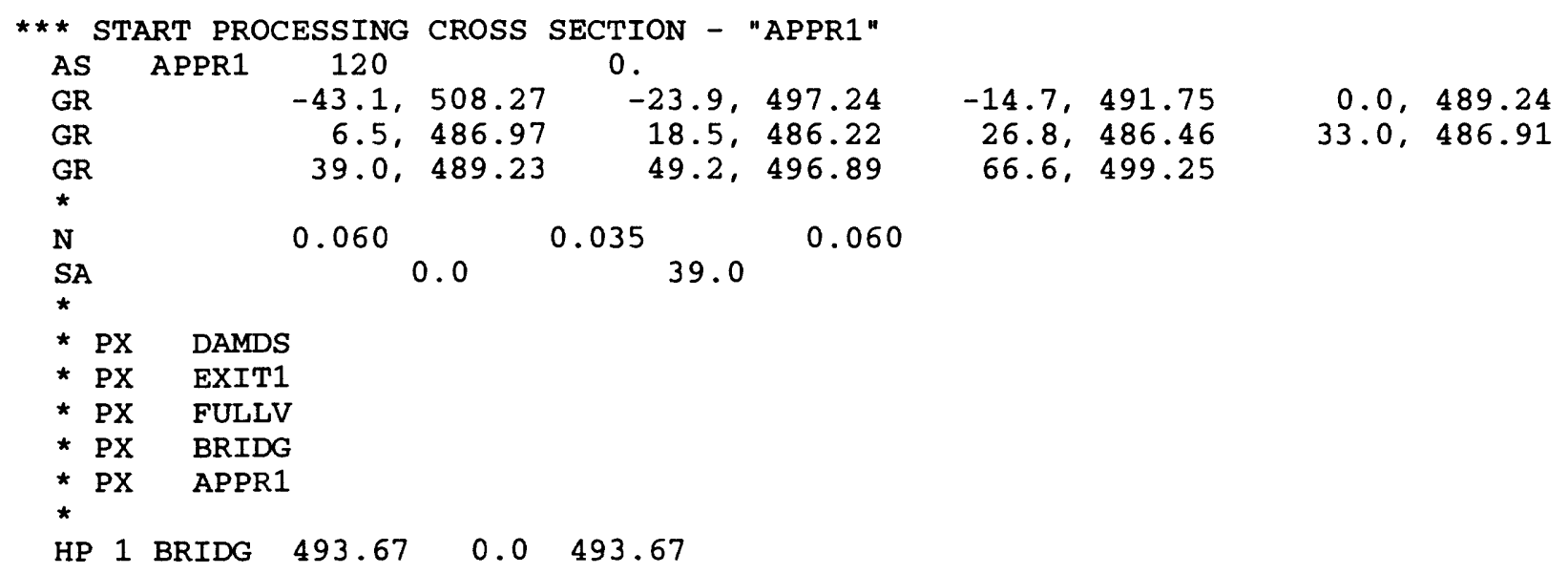

$-14.7,491.75$

$26.8,486.46$

$66.6,499.25$

$0.0,489.24$

$33.0,486.91$

GR

*
N
0.060
0.0
0.035
39.0
0.060

$\star$

* PX DAMDS

* PX EXIT1

* PX FULLV

* PX BRIDG

* PX APPR 1

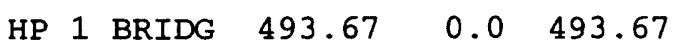

*** FINISH PROCESSING CROSS SECTION - "APPR1"

* * CROSS SECTION "APPR1" WRITTEN TO DISK, RECORD NO. = 7

--- DATA SUMMARY FOR SECID "APPR1" AT SRD $=120$. ERR-CODE $=$ 
$\begin{array}{rrrrr}\text { SKEW } & \text { IHFNO } & \text { VSLOPE } & \text { EK } & \mathrm{CK} \\ 0.0 & 0 . & 0.0002 & 0.50 & 0.00\end{array}$

$X-Y$ COORDINATE PAIRS (NGP $=11)$ :

$\begin{array}{rrrrrrrr}X & Y & X & Y & X & Y & X & Y \\ -43.1 & 508.27 & -23.9 & 497.24 & -14.7 & 491.75 & 0.0 & 489.24 \\ 6.5 & 486.97 & 18.5 & 486.22 & 26.8 & 486.46 & 33.0 & 486.91 \\ 39.0 & 489.23 & 49.2 & 496.89 & 66.6 & 499.25 & & \end{array}$

$X-Y$ MAX-MIN POINTS:

$\begin{array}{rrrrrrrr}\text { XMIN } & Y & X & \text { YMIN } & \text { XMAX } & Y & X & \text { YMAX } \\ -43.1 & 508.27 & 18.5 & 486.22 & 66.6 & 499.25 & -43.1 & 508.27\end{array}$

SUBAREA BREAKPOINTS (NSA $=3$ ):

0.39 .

ROUGHNESS COEFFICIENTS (NSA $=3)$ :

$$
\begin{array}{lll}
0.060 & 0.035 & 0.060
\end{array}
$$

$\begin{array}{llll}66.6 & 499.25 & -43.1 & 508.27\end{array}$

BRIDGE PROJECTION DATA: XREFLT XREFRT FDSTLT FDSTRT

1

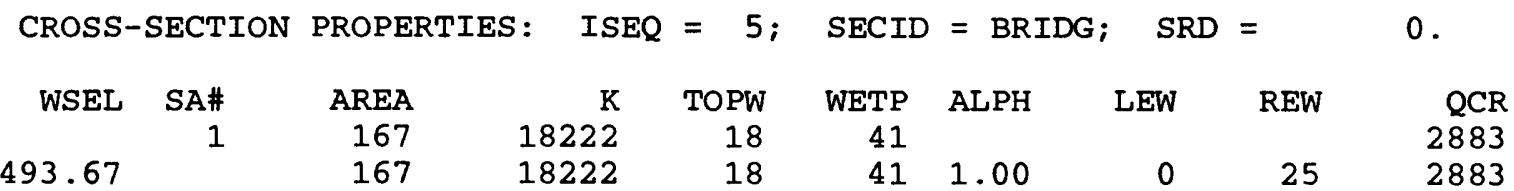

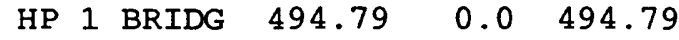

\begin{tabular}{rrrrrrrrrr}
\multicolumn{2}{l}{ CROSS-SECTION } & PROPERTIES: & \multicolumn{2}{c}{ ISEQ $=$} & $5 ;$ & SECID $=$ BRIDG; & SRD $=$ & 0. \\
WSEL & SA\# & AREA & K & TOPW & WETP & ALPH & LEW & REW & QCR \\
& 1 & 185 & 20602 & 15 & 44 & & & & 3654 \\
494.79 & & 185 & 20602 & 15 & 44 & 1.00 & 0 & 25 & 3654
\end{tabular}

HP 1 BRIDG $495.30 \quad 0.0 \quad 495.30$

CROSS-SECTION PROPERTIES: ISEQ $=5 ;$ SECID $=$ BRIDG $;$ SRD $=0$.

$\begin{array}{rrrrrrrrrr}\text { WSEL } & \text { SA\# } & \text { AREA } & \text { K } & \text { TOPW } & \text { WETP } & \text { ALPH } & \text { LEW } & \text { REW } & \text { QCR } \\ & 1 & 193 & 21412 & 14 & 46 & & & & 4086 \\ 495.30 & & 193 & 21412 & 14 & 46 & 1.00 & 0 & 25 & 4086\end{array}$

HP 1 BRIDG $\quad 495.44 \quad 0.0 \quad 495.44$

CROSS-SECTION PROPERTIES: ISEQ $=5 ;$ SECID $=$ BRIDG; SRD $=0$.

$\begin{array}{rrrrrrrrrr}\text { WSEL } & \text { SA\# } & \text { AREA } & \text { K } & \text { TOPW } & \text { WETP } & \text { ALPH } & \text { LEW } & \text { REW } & \text { QCR } \\ & 1 & 195 & 21495 & 13 & 47 & & & & 4270 \\ 495.44 & & 195 & 21495 & 13 & 47 & 1.00 & 0 & 25 & 4270\end{array}$

HP 1 BRIDG $\quad 497.14 \quad 0.0 \quad 497.14$

CROSS-SECTION PROPERTIES: ISEQ $=5 ;$ SECID $=$ BRIDG; $\quad$ SRD $=0$.

$\begin{array}{rrrrrrrrrr}\text { WSEL } & \text { SA\# } & \text { AREA } & \text { K } & \text { TOPW } & \text { WETP } & \text { ALPH } & \text { LEW } & \text { REW } & \text { QCR } \\ & 1 & 206 & 19905 & 0 & 60 & & & & 0 \\ 97.14 & & 206 & 19905 & 0 & 60 & 1.00 & 0 & 25 & 0\end{array}$

1

497.14

$206 \quad 19905$

$60 \quad 1.00$

0
0

HP 1 APPR1 $494.23 \quad 0.0 \quad 494.23$

CROSS-SECTION PROPERTIES: ISEQ $=7 ;$ SECID $=$ APPRI; SRD $=120$. 


$\begin{array}{rrrrrrrrrr}\text { WSEL } & \text { SA\# } & \text { AREA } & \text { K } & \text { TOPW } & \text { WETP } & \text { ALPH } & \text { LEW } & \text { REW } & \text { QCR } \\ & 1 & 60 & 3130 & 19 & 20 & & & & 608 \\ & 2 & 281 & 43892 & 39 & 40 & & & & 4272 \\ 494.23 & 3 & 17 & 656 & 7 & 8 & & & & 149 \\ & & 357 & 47678 & 65 & 68 & 1.28 & -18 & 46 & 4225\end{array}$

HP 1 APPR $1 \quad 496.04 \quad 0.0 \quad 496.04$

CROSS-SECTION PROPERTIES: ISEQ $=7 ;$ SECID $=$ APPR1; $\quad$ SRD $=120$.

$\begin{array}{rrrrrrrrrr}\text { WSEL } & \text { SA\# } & \text { AREA } & \text { K } & \text { TOPW } & \text { WETP } & \text { ALPH } & \text { LEW } & \text { REW } & \text { QCR } \\ & 1 & 97 & 6229 & 22 & 23 & & & & 1157 \\ & 2 & 351 & 63794 & 39 & 40 & & & & 5982 \\ & 3 & 31 & 1495 & 9 & 11 & & & & 323 \\ 96.04 & & 479 & 71518 & 70 & 74 & 1.34 & -21 & 48 & 6149\end{array}$

HP 1 APPR $1 \quad 497.22 \quad 0.0 \quad 497.22$

CROSS-SECTION PROPERTIES: ISEQ $=7 ;$ SECID $=$ APPR $1 ; \quad$ SRD $=120$.

WSEL SA\# AREA $\mathrm{K}$ TOPW WETP ALPH LEW REW

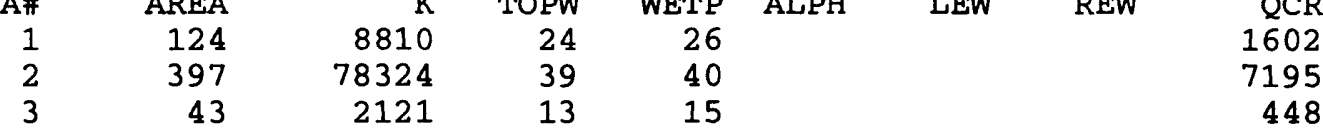

497.22

$564 \quad 89255 \quad 75$

$81 \quad 1.38 \quad-23 \quad 52$

7435

HP 1 APPR $1 \quad 497.66 \quad 0.0 \quad 497.66$

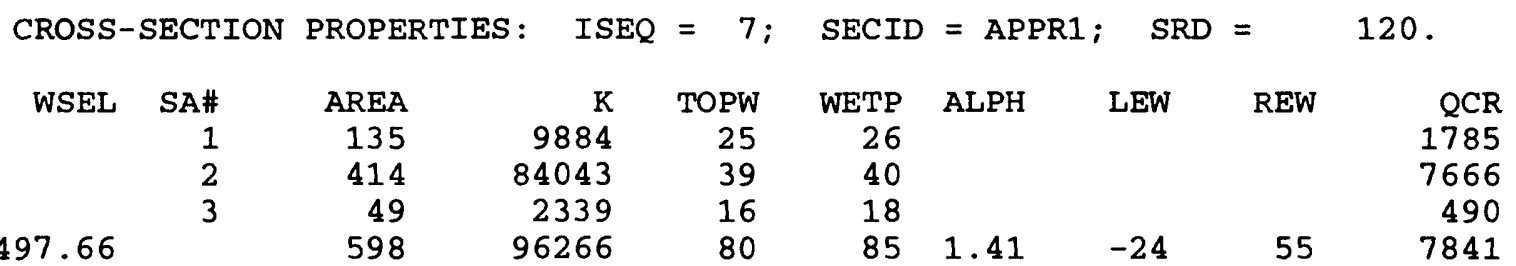

$\begin{array}{llll}\text { HP } 1 \text { APPR } 1 & 500.02 & 0.0 & 500.02\end{array}$

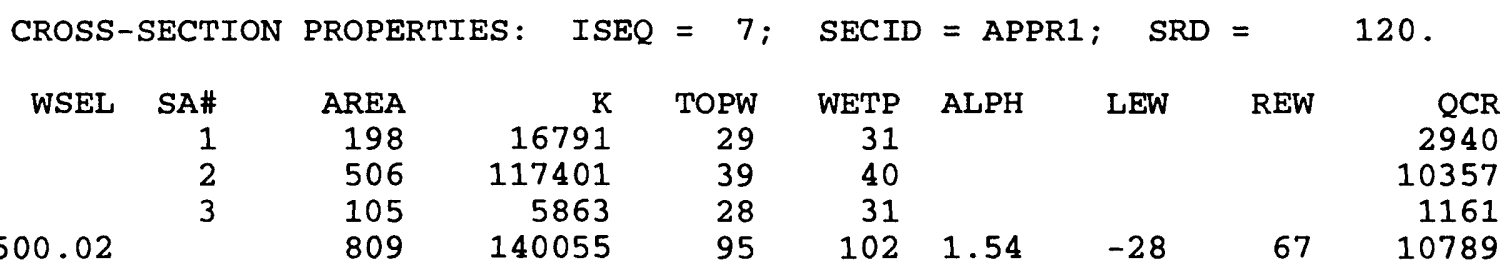

1

*

EX

+++ BEGINNING PROFILE CALCULATIONS -- 6

$\begin{array}{rrrrrrrrrr}\text { XSID : CODE } & \text { SRDL } & \text { LEW } & \text { AREA } & \text { VHD } & \text { HF } & \text { EGL } & \text { CRWS } & Q & \text { WSEL } \\ \text { SRD } & \text { FLEN } & \text { REW } & \text { K } & \text { ALPH } & \text { HO } & \text { ERR } & \text { FR\# } & \text { VEL } & \end{array}$

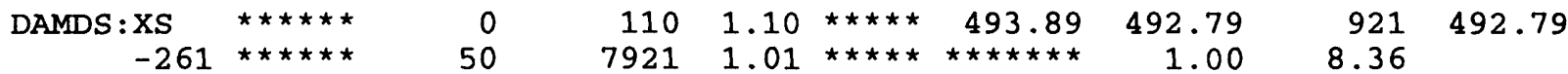

$===135$ CONVEYANCE RATIO OUTSIDE OF RECOMMENDED LIMITS.

"EXIT2" KRATIO $=6.94$

$\begin{array}{crrrrrrrrr}\text { EXIT2 : XS } & 1 & -11 & 387 & 0.10 & 0.00 & 493.89 & \star \star \star \star \star \star \star & 921 & 493.79 \\ -260 & 1 & 55 & 54996 & 1.13 & 0.00 & 0.00 & 0.19 & 2.38 & \\ \text { EXIT1 : XS } & 188 & -11 & 393 & 0.10 & 0.05 & 493.94 & & & \\ -72 & 188 & 55 & 56492 & 1.20 & 0.00 & 0.00 & 0.19 & 2.34 & 493.84\end{array}$




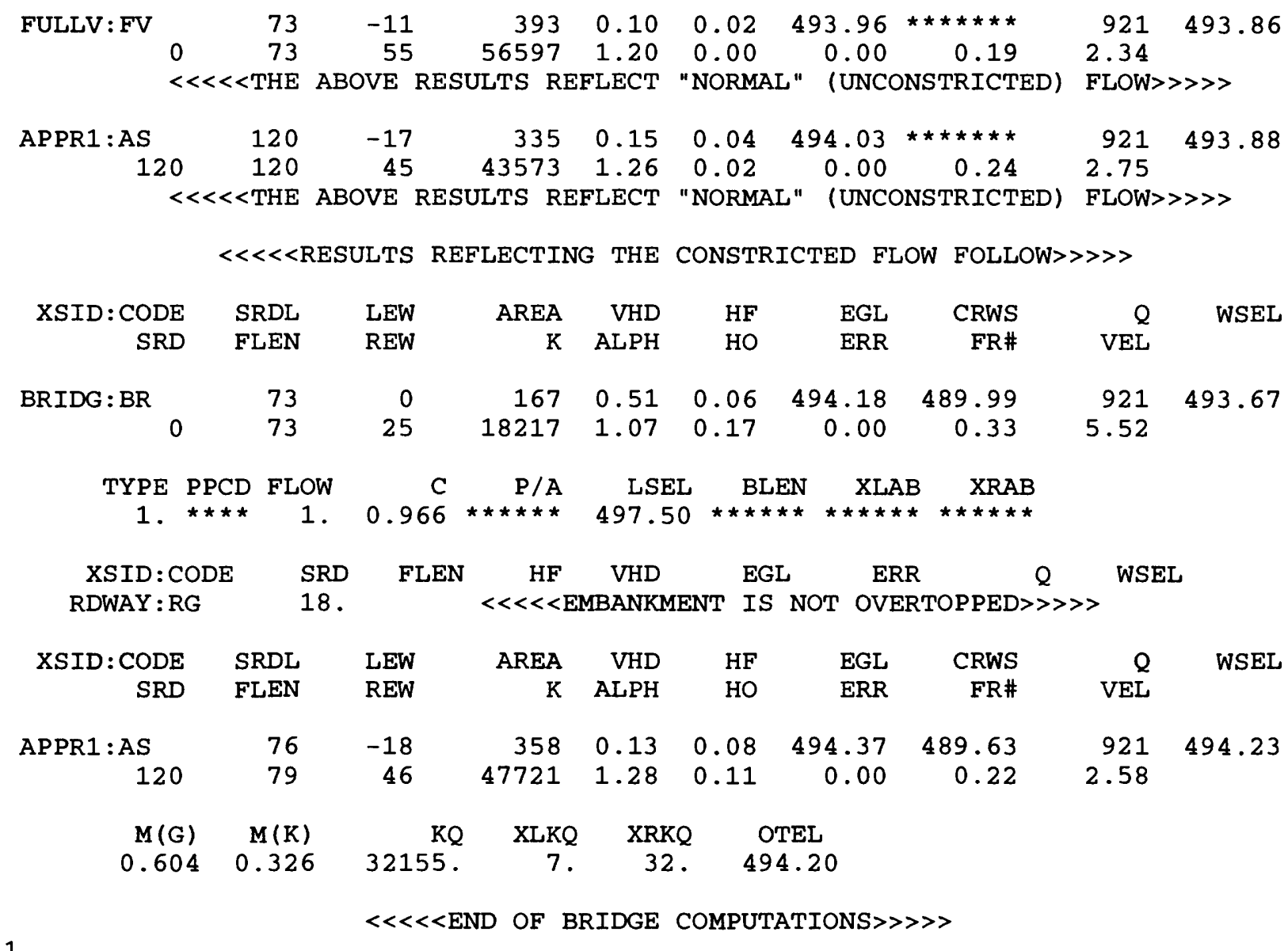

FIRST USER DEFINED TABLE.

\begin{tabular}{|c|c|c|c|c|c|c|c|c|}
\hline XSID:CODE & SRD & LEW & REW & $Q$ & K & AREA & VEL & WSEL \\
\hline DAMDS : XS & -262 & 0 . & 50. & 921. & 7921 . & 110 & 8.36 & 492.79 \\
\hline EXIT2 : XS & -261 & -12 & 55. & 921. & 54996 . & 387. & 2.38 & 493.79 \\
\hline EXIT1:XS & -73 & -12 & 55. & 921. & 56492 . & 393. & 2.34 & 493.84 \\
\hline FULLV : FV & 0 . & -12 & 55. & 921. & 56597 . & 393. & 2.34 & 493.86 \\
\hline BRIDG : BR & 0 . & 0 & 25 . & 921. & 18217 . & 167 . & 5.52 & 493.67 \\
\hline RDWAY : RG & 18. & $* \star \star \star \star \star x$ & $\star \star \star \star$ & 0 . & 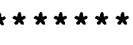 & $\star \star \star \star \star *$ & 1.00 & $\star \star \star \star \star *$ \\
\hline APPR1 : AS & 120 . & -19 & 46. & 921. & 47721 . & 358 . & 2.58 & 494.23 \\
\hline $\begin{array}{l}\text { XSID : CODE } \\
\text { APPR1 : AS }\end{array}$ & $\begin{array}{r}\text { XLKQ } \\
7 .\end{array}$ & $\begin{array}{l}\text { XRKQ } \\
32 .\end{array}$ & 321 & & & & & \\
\hline
\end{tabular}

SECOND USER DEFINED TABLE.

\begin{tabular}{|c|c|c|c|c|c|c|c|c|c|}
\hline XSID:CODE & CRWS & FR\# & YMIN & YMAX & $\mathrm{HF}$ & $\mathrm{HO}$ & VHD & EGL & EL \\
\hline DAMDS : XS & 492.79 & 1.00 & 90.58 & 08.27 & & 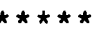 & 1.10 & 493.89 & 20 \\
\hline XIT2: XS & 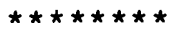 & 0.19 & 485.15 & 502.88 & 0.00 & 0.00 & & y & 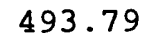 \\
\hline XIT1: XS & 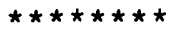 & 0.19 & 485.11 & 202. & 0 . & & & 4 & \\
\hline$V: F V$ & 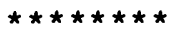 & 0. & 485.1 & 502. & 0 . & 0 & 0 & 3. & \\
\hline $\mathrm{BR}$ & 489.99 & 0 . & & .14 & 0.06 & 0.17 & 0.51 & 494.18 & I \\
\hline : RG & & & & 27 & & & & $\star \star \star$ & \\
\hline : AS & 489.63 & 0.22 & 486.22 & 508.27 & 0.08 & 0.11 & 0.13 & 494.37 & 494 \\
\hline
\end{tabular}

1

\begin{tabular}{|c|c|c|c|c|c|c|c|c|c|}
\hline XSID: CODE & SRDL & LEW & AREA & VHD & HF & EGL & CRWS & & WSEL \\
\hline SRD & FLEN & REW & $\mathrm{K}$ & ALPH & HO & ERR & FR\# & VEL & \\
\hline $\begin{aligned} A M D S: & X S \\
& -261\end{aligned}$ & 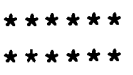 & $\begin{array}{r}-20 \\
60\end{array}$ & $\begin{array}{r}180 \\
16513\end{array}$ & $\begin{array}{l}1.28 \\
1.10\end{array}$ & $\begin{array}{l}\star \star \star \star \star \\
\star \star \star \star \star\end{array}$ & $\begin{array}{r}495.28 \\
\star \star \star \star \star \star \star \star\end{array}$ & $\begin{array}{r}493.73 \\
1.07\end{array}$ & $\begin{array}{l}1553 \\
8.65\end{array}$ & 49 \\
\hline
\end{tabular}




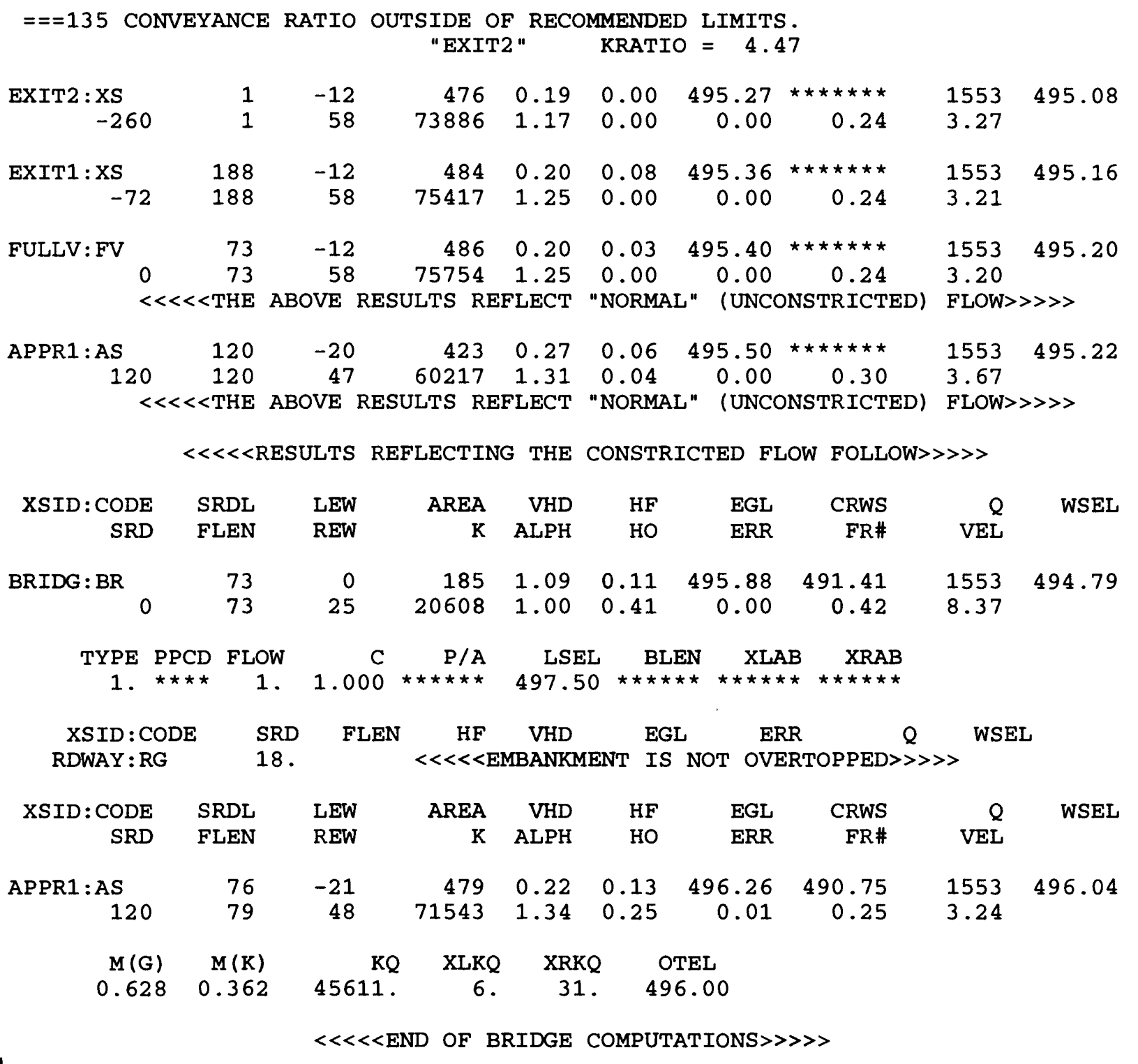

FIRST USER DEFINED TABLE.

\begin{tabular}{|c|c|c|c|c|c|c|c|c|}
\hline XSID:CODE & SRD & LEW & REW & $Q$ & $\mathrm{~K}$ & AREA & VEL & WSEL \\
\hline DAMDS : XS & -262 & -21 & 60. & 1553. & 16513 . & 180. & 8.65 & 494.00 \\
\hline EXIT2:XS & -261 & -13 & 58. & 1553. & 73886 . & 476 . & 3.27 & 495.08 \\
\hline EXIT1:XS & -73 & -13 & 58. & 1553. & 75417 . & 484 . & 3.21 & 495.16 \\
\hline FULLV : FV & 0 & -13 & 58. & 1553. & 75754 . & 486 . & 3.20 & 495.20 \\
\hline BRIDG : BR & 0 & 0. & 25 . & 1553. & 20608 & 185. & 8.37 & 494.79 \\
\hline RDWAY : RG & 18 & $\star \star \star \star \star \star *$ & $\star \star \star \star \star$ & 0 . & $\star \star \star \star \star$ & $\star \star \star$ & 1.00 & 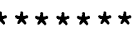 \\
\hline APPR1: AS & 120. & -22 & 48. & 1553 . & 71543. & 479 . & 3.24 & 496.04 \\
\hline SID : CODE & XLKQ & $\mathrm{XRKQ}$ & & & & & & \\
\hline APPR1:AS & 6 & 31. & & & & & & \\
\hline
\end{tabular}

SECOND USER DEFINED TABLE.

\begin{tabular}{|c|c|c|c|c|c|c|c|c|c|}
\hline XSID: CODE & CRWS & FR\# & YMIN & YMAX & HF & HO & VHD & EGL & WSEL \\
\hline DAMDS : XS & 93.73 & 1.07 & 490.58 & $27 *$ & + & $* \star *$ & 1.28 & 495.2 & 494.00 \\
\hline $2: x S$ & 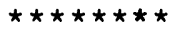 & 0.2 & 485 & 502.88 & 0.00 & 0.00 & 0 & 7 & 4 \\
\hline EXIT1:XS & 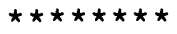 & 0.24 & 485.11 & 502.84 & 0.08 & 0.00 & 0.20 & 495.36 & 495 \\
\hline
\end{tabular}




\begin{tabular}{|c|c|c|c|c|c|c|c|c|c|}
\hline & $\star \star$ & & 12 & 5 & 3 & 0 & 0 & 0 & \\
\hline & & 0 & & & 0.11 & 0 & 1.09 & 38 & \\
\hline & $\begin{array}{l}\star \star \star \star \star \star \star \star \star \\
490.75\end{array}$ & $\begin{array}{l}* \star \star \star \star \\
0.25\end{array}$ & & & $\begin{array}{l}\star \star \star \star \star \star \star \star ~ \\
0.13\end{array}$ & $\begin{array}{l}\star \star \star \star \star \star \\
0.25\end{array}$ & 0.22 & & \\
\hline
\end{tabular}

\begin{tabular}{|c|c|c|c|c|c|c|c|c|c|}
\hline XSID: CODE & SRDL & LEW & AREA & VHD & $\mathrm{HF}$ & EGL & CRWS & $Q$ & WSEL \\
\hline SRD & FLEN & REW & $\mathrm{K}$ & ALPH & HO & ERR & FR\# & VEL & \\
\hline $\begin{aligned} \text { AMDS }: & \text { XS } \\
& -261\end{aligned}$ & $\begin{array}{l}\star \star \star \star \star \star \star \star \\
\star \star \star \star \star \star \star \star\end{array}$ & $\begin{array}{r}-28 \\
63\end{array}$ & $\begin{array}{r}230 \\
21871\end{array}$ & $\begin{array}{l}1.44 \\
1.23\end{array}$ & $\begin{array}{l}\star \star \star \star \star \star \\
\star \star \star \star \star \star\end{array}$ & $\begin{array}{r}496.02 \\
\star \star \star \star \star \star \star \star \star ~\end{array}$ & $\begin{array}{r}494.43 \\
1.08\end{array}$ & $\begin{array}{l}1989 \\
8.66\end{array}$ & 8 \\
\hline
\end{tabular}

$===135$ CONVEYANCE RATIO OUTSIDE OF RECOMMENDED LIMITS .

" EXIT2" KRATIO $=3.87$

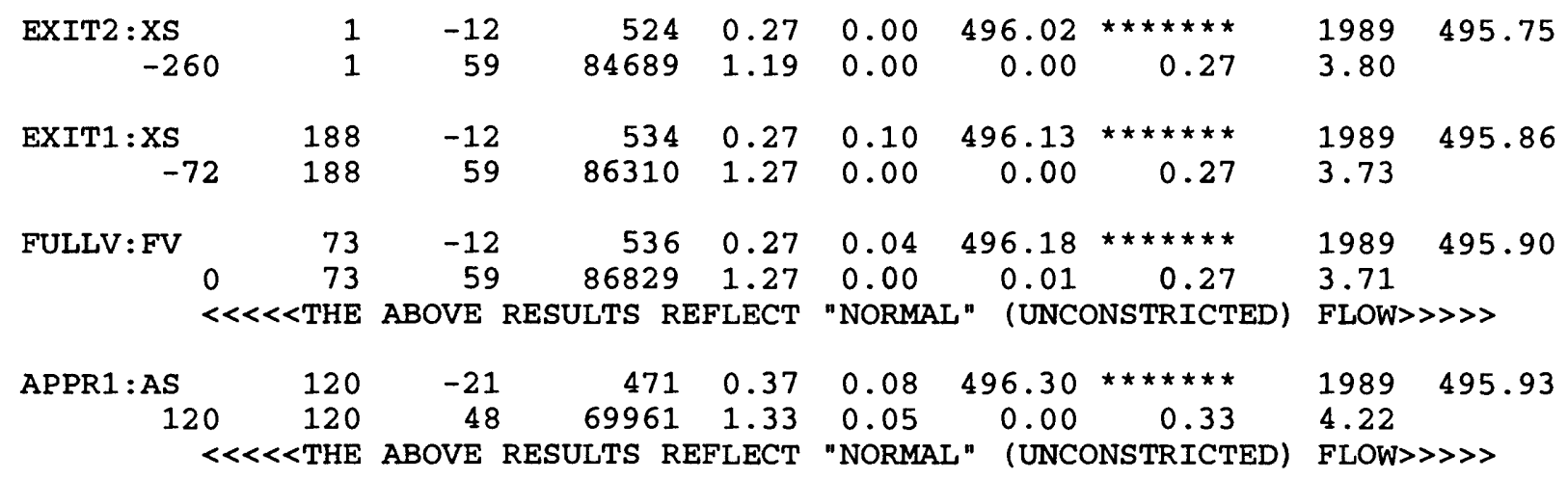

$\ll<<<$ RESULTS REFLECTING THE CONSTRICTED FLOW FOLLOW $\gg \gg \gg>$

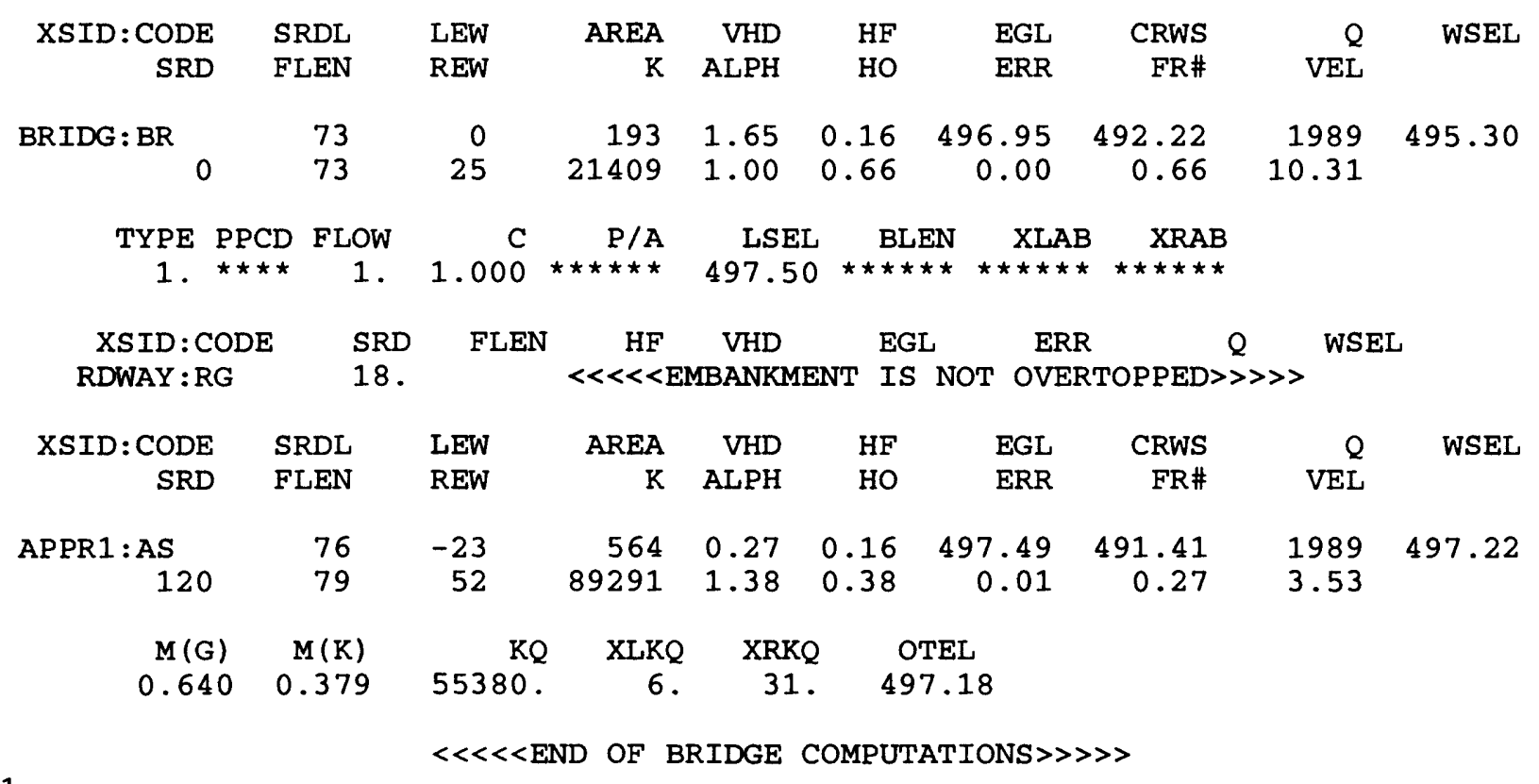

FIRST USER DEFINED TABLE.

\begin{tabular}{|c|c|c|c|c|c|c|c|c|}
\hline XSID:CODE & SRD & LEW & REW & Q & $\mathrm{K}$ & AREA & VEL & WSEL \\
\hline DAMDS : XS & -262 . & -29 & 63. & 1989. & 21871 . & 230 & 8.66 & 494.58 \\
\hline EXIT2:XS & -261 & -13 & 59 & 1989. & 84689 . & 524 . & 3.80 & 495.75 \\
\hline EXIT1:XS & -73 & -13 & 59 & 1989. & 86310. & 534. & 3.73 & 495.86 \\
\hline FULLV:FV & 0. & -13 & 59 & 1989. & 29. & 53 & 3.71 & 495.90 \\
\hline BRIDG : BR & 0 . & 0 . & 25. & 1989. & 21409 . & 193. & 10.31 & 495.30 \\
\hline RDWAY : RG & 18. & $\star \star \star \star \star$ & $\star \star \star \star$ & & & $\star \star \star \star \star \star$ & 1.00 & $\star \star \star \star \star \star \star \star \star ~$ \\
\hline APPR $1:$ AS & 120 & -24 & 52. & 1989. & 89291. & 564. & 3.53 & 497.22 \\
\hline
\end{tabular}




$\begin{array}{cccr}\text { XSID: CODE } & \text { XLKQ } & \text { XRKQ } & \text { KQ } \\ \text { APPR1:AS } & 6 . & 31 . & 55380 .\end{array}$

SECOND USER DEFINED TABLE.

\begin{tabular}{|c|c|c|c|c|c|c|c|c|c|}
\hline XSID:CO & CRWS & FR\# & YMIN & YMAX & $\mathrm{HF}$ & HO & VHD & EGL & WSEL \\
\hline DAMDS : XS & 43 & 1.08 & 490. & 50 & & & 44 & 496 & 49 \\
\hline EXIT2:XS & & 0 & & & 0.00 & 0.00 & & & \\
\hline EXI & $\star \star \star \star \star \star \star \star \star$ & 0 & & & & & & & \\
\hline $\mathrm{FU}$ & 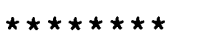 & 0.27 & & & 0 . & 0 & & & \\
\hline BRII & 492.22 & 0.6 & 486 & 4 & 0.16 & 0.66 & 1.65 & 496.95 & .30 \\
\hline RDWAY : RG & & $\star \star$ & & & & & & & $\star \star \star$ \\
\hline APP & 491.41 & 0.27 & & & 0.16 & 0.38 & 0.27 & 497.49 & 497.22 \\
\hline
\end{tabular}

$==015$ WSI IN WRONG FLOW REGIME AT SECID "DAMDS": USED WSI = CRWS .

$$
\text { WSI, CRWS }=494.28 \quad 494.64
$$

\begin{tabular}{|c|c|c|c|c|c|c|c|c|c|}
\hline XSID: CODE & SRDL & LEW & AREA & VHD & $\mathrm{HF}$ & EGL & CRWS & $Q$ & WSEL \\
\hline SRD & FLEN & REW & $\mathrm{K}$ & ALPH & HO & ERR & FR\# & VEL & \\
\hline $\begin{aligned} \text { MDS : } & X S \\
& -261\end{aligned}$ & $\begin{array}{l}\star \star \star \star \star \star \star \\
\star \star \star \star \star \star \star \star ~\end{array}$ & $\begin{array}{r}-29 \\
64\end{array}$ & $\begin{array}{r}235 \\
22503\end{array}$ & $\begin{array}{l}1.62 \\
1.25\end{array}$ & $\begin{array}{l}\star \star \star \star \star \star \\
\star \star \star \star \star \star\end{array}$ & $\begin{array}{r}496.26 \\
\star \star \star \star \star \star \star \star \star\end{array}$ & $\begin{array}{r}494.64 \\
1.13\end{array}$ & $\begin{array}{l}2150 \\
9.13\end{array}$ & 4 \\
\hline
\end{tabular}

$===135$ CONVEYANCE RATIO OUTSIDE OF RECOMMENDED LIMITS .

" EXIT2" KRATIO = 3.92

\begin{tabular}{|c|c|c|c|c|c|c|c|c|c|}
\hline $\begin{array}{rl}\text { EXIT2 } 2 & X S \\
& -260\end{array}$ & $\begin{array}{l}1 \\
1\end{array}$ & $\begin{array}{r}-12 \\
60\end{array}$ & $\begin{array}{r}539 \\
88248\end{array}$ & $\begin{array}{l}0.30 \\
1.19\end{array}$ & $\begin{array}{l}0.00 \\
0.00\end{array}$ & $\begin{array}{r}496.26 \\
0.00\end{array}$ & 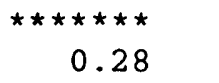 & $\begin{array}{l}2150 \\
3.99\end{array}$ & 495.97 \\
\hline $\begin{array}{r}\text { EXIT1: XS } \\
-72\end{array}$ & $\begin{array}{l}188 \\
188\end{array}$ & $\begin{array}{r}-12 \\
60\end{array}$ & $\begin{array}{r}550 \\
89922\end{array}$ & $\begin{array}{l}0.30 \\
1.28\end{array}$ & $\begin{array}{l}0.11 \\
0.00\end{array}$ & $\begin{array}{r}496.38 \\
0.00\end{array}$ & $\begin{array}{r}\star \star \star \star \star \star \star \star \star ~ \\
0.28\end{array}$ & $\begin{array}{l}2150 \\
3.91\end{array}$ & 196.08 \\
\hline ULLV : FV & $\begin{array}{l}73 \\
73\end{array}$ & $\begin{array}{r}-12 \\
60\end{array}$ & $\begin{array}{r}552 \\
90513\end{array}$ & $\begin{array}{l}0.30 \\
1.28\end{array}$ & $\begin{array}{l}0.04 \\
0.00\end{array}$ & $\begin{array}{r}496.43 \\
0.01\end{array}$ & 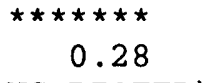 & $\begin{array}{l}2150 \\
3.89\end{array}$ & 496.13 \\
\hline
\end{tabular}

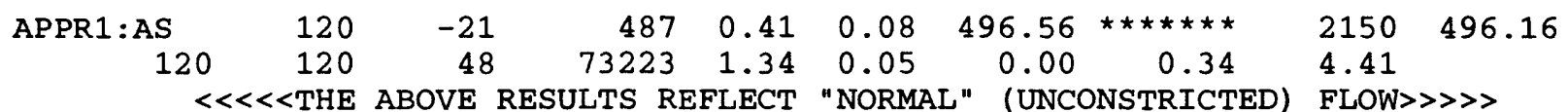

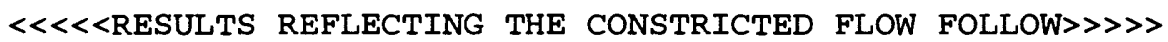

\begin{tabular}{|c|c|c|c|c|c|c|c|c|c|}
\hline XSID: CODE & SRDL & LEW & AREA & VHD & $\mathrm{HF}$ & EGL & CRWS & Q & WSEL \\
\hline SRD & FLEN & REW & $\mathrm{K}$ & ALPH & HO & ERR & FR\# & VEL & \\
\hline $\mathrm{BR}$ & $\begin{array}{l}73 \\
73\end{array}$ & $\begin{array}{r}0 \\
25\end{array}$ & $\begin{array}{r}195 \\
21495\end{array}$ & $\begin{array}{l}1.89 \\
1.00\end{array}$ & $\begin{array}{l}0.17 \\
0.78\end{array}$ & $\begin{array}{r}497.33 \\
0.00\end{array}$ & $\begin{array}{r}492.49 \\
0.70\end{array}$ & $\begin{array}{r}2150 \\
11.04\end{array}$ & 4 \\
\hline
\end{tabular}

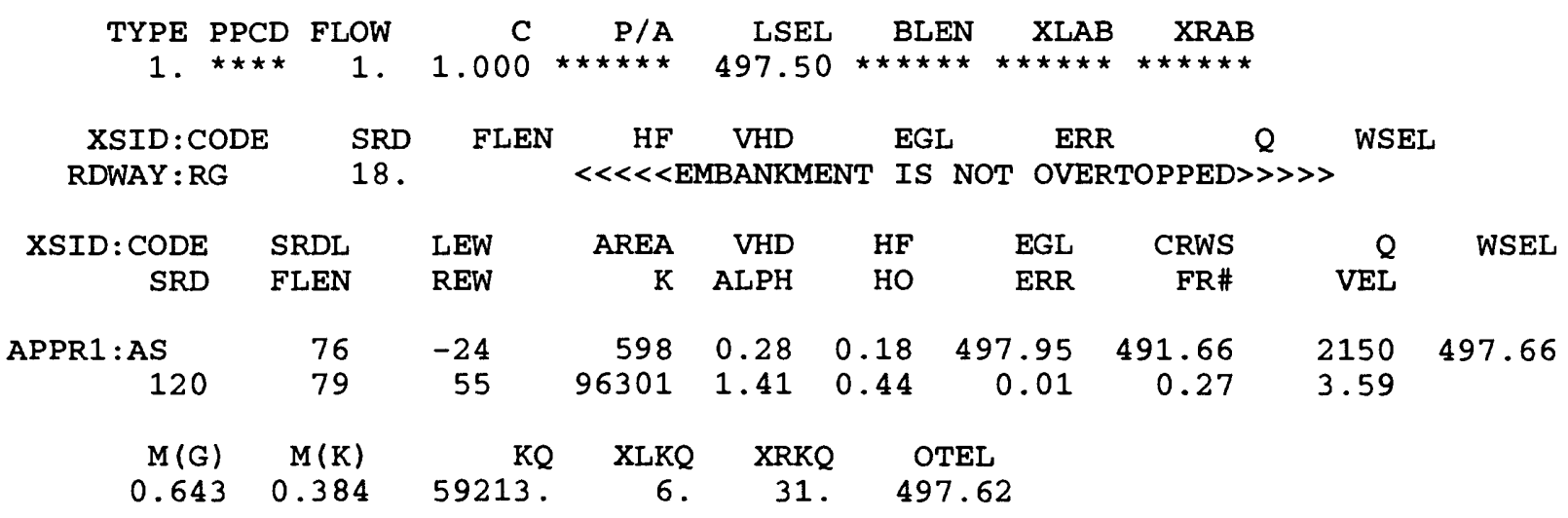

$\ll<<<$ END OF BRIDGE COMPUTATIONS $\gg \gg>>>$ 
FIRST USER DEFINED TABLE.

\begin{tabular}{|c|c|c|c|c|c|c|c|c|}
\hline XSID:CODE & SRD & LEW & REW & 8 & $\mathrm{~K}$ & AREA & VEL & WSEL \\
\hline DAMDS : XS & -262 . & -30 & 64. & 2150 & 22503 & 235 . & 9.13 & 494.64 \\
\hline EXIT2:XS & -261 & -13 & 60. & 2150 & 88248 . & 539. & 3.99 & 495.97 \\
\hline EXIT1:XS & -73. & -13 & 60. & 2150 & 89922 . & 550 . & 3.91 & 496.08 \\
\hline FULLV:FV & 0 . & -13 & 60. & 2150 & 90513. & 552 . & 3.89 & 496.13 \\
\hline BRIDG : BR & 0 . & 0 . & 25 . & 2150 & 21495 & 195. & 11.04 & 495.44 \\
\hline RDWAY : RG & 18. & $\star \star \star \star \star \star$ & $\star \star \star \star \star$ & 0. & $\star \star \star$ & $\star \star \star \star \star \star$ & 1.00 & 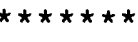 \\
\hline APPR1: AS & 120. & -25 & 55. & 2150 & 96301. & 598 . & 3.59 & 497.66 \\
\hline XSID : CODE & XLKQ & $\mathrm{XRKQ}$ & & & & & & \\
\hline APPR 1:AS & 6 . & 31. & 5921 & & & & & \\
\hline
\end{tabular}

SECOND USER DEFINED TABLE.

1

\begin{tabular}{|c|c|c|c|c|c|c|c|c|c|}
\hline XSID: CODE & CRWS & FR\# & YMIN & YMAX & $\mathrm{HF}$ & 0 & VHD & EGL & WSEL \\
\hline AMDS : XS & 494 & 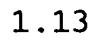 & 190 & 50 & & 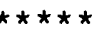 & & 496. & \\
\hline$S$ & $x \times x \times x \times$ & $.25+2$ & $8=$ & 8 & 0.00 & 0.00 & & & \\
\hline is & $\star \star \star \star \star \star \star$ & & & & & & & & \\
\hline $\mathrm{V}$ & 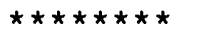 & & & & & & & & \\
\hline & 402 & & & & 0.17 & 0.78 & 1. & & \\
\hline & & $\star \star \star \star$ & & & & & & & \\
\hline PPR1:AS & 491.66 & 0.27 & & & 0.18 & 0.44 & 0.28 & 497.95 & 4976 \\
\hline
\end{tabular}

$==015$ WSI IN WRONG FLOW REGIME AT SECID "DAMDS": USED WSI = CRWS.

WSI, CRWS $=494.28 \quad 494.66$

$\begin{array}{rrrrrrrrrr}\text { XSID : CODE } & \text { SRDL } & \text { LEW } & \text { AREA } & \text { VHD } & \text { HF } & \text { EGL } & \text { CRWS } & \text { Q } & \text { WSEL } \\ \text { SRD } & \text { FLEN } & \text { REW } & \text { K } & \text { ALPH } & \text { HO } & \text { ERR } & \text { FR\# } & \text { VEL } & \\ & & & & & & & & & \\ \text { DAMDS : XS } & \star \star \star \star \star \star & -29 & 237 & 1.62 & \star \star \star \star \star * & 496.28 & 494.66 & 2160 & 494.66 \\ -261 & \star \star \star \star \star \star & 64 & 22707 & 1.25 * \star \star \star \star \star * \star * \star \star * \star * & 1.13 & 9.10 & \end{array}$

$===135$ CONVEYANCE RATIO OUTSIDE OF RECOMMENDED LIMITS.

"EXIT2" KRATIO $=3.90$

\begin{tabular}{|c|c|c|c|c|c|c|c|c|c|}
\hline $\begin{aligned} X I T 2: & X S \\
& -260\end{aligned}$ & $\begin{array}{l}1 \\
1\end{array}$ & $\begin{array}{r}-12 \\
60\end{array}$ & $\begin{array}{r}540 \\
88470\end{array}$ & $\begin{array}{l}0.30 \\
1.19\end{array}$ & $\begin{array}{l}0.00 \\
0.00\end{array}$ & $\begin{array}{r}496.28 \\
0.00\end{array}$ & 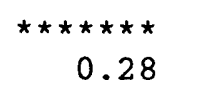 & $\begin{array}{l}2160 \\
4.00\end{array}$ & 495.98 \\
\hline $\begin{array}{r}\text { XIT1: XS } \\
-72\end{array}$ & $\begin{array}{l}188 \\
188\end{array}$ & $\begin{array}{r}-12 \\
60\end{array}$ & $\begin{array}{r}551 \\
90147\end{array}$ & 1. & & $\begin{array}{r}496.40 \\
0.00\end{array}$ & $\begin{array}{r}\star \star \star \star \star \star \star \star \star \\
0.28\end{array}$ & & 49 \\
\hline JLLV:FV & $\begin{array}{r}73 \\
73 \\
\text { THE }\end{array}$ & $\begin{array}{r}-12 \\
60 \\
\text { ABOVE }\end{array}$ & $\begin{array}{r}553 \\
90744 \\
\text { SULTS R. }\end{array}$ & $\begin{array}{r}0.30 \\
1.28 \\
\text { FLECT }\end{array}$ & $\begin{array}{l}0.04 \\
0.00 \\
\text { "NORMAI }\end{array}$ & $\begin{array}{r}496.45 \\
0.01 \\
\text { (UNCC }\end{array}$ & $\begin{array}{c}\star \star \star \star \star \star \star \star \star \\
0.28 \\
\text { ONSTRICTED) }\end{array}$ & $\begin{array}{l}2160 \\
3.90 \\
\text { FLOW> }\end{array}$ & 4 \\
\hline PR1: AS & $\begin{array}{r}120 \\
120 \\
<\mathrm{THE}\end{array}$ & $\begin{array}{r}-21 \\
48 \\
\text { OVE }\end{array}$ & $\begin{array}{r}488 \\
73427 \\
\text { JLTS R }\end{array}$ & $\begin{array}{r}0.41 \\
1.34 \\
\text { FLECT }\end{array}$ & $\begin{array}{l}0.08 \\
0.05 \\
\text { NORMAI }\end{array}$ & $\begin{array}{r}496.58 \\
0.00 \\
\text { [UNCC }\end{array}$ & $\begin{array}{c}\star \star \star \star \star \star \star \star \star ~ \\
0.34 \\
\text { ONSTRICTE }\end{array}$ & $\begin{array}{l}2160 \\
4.42 \\
\text { FLOW }\end{array}$ & .17 \\
\hline
\end{tabular}

$==220$ FLOW CLASS 1 (4) SOLUTION INDICATES POSSIBLE PRESSURE FLOW.

$$
\text { WS3,WSIU,WS1, LSEL }=\begin{array}{llll}
495.45 & 497.51 & 497.69 & 497.50
\end{array}
$$

$==245$ ATTEMPTING FLOW CLASS 2 (5) SOLUTION .

$\ll<<<$ RESULTS REFLECTING THE CONSTRICTED FLOW FOLLOW $\gg \gg>>$

\begin{tabular}{|c|c|c|c|c|c|c|c|c|c|}
\hline $\begin{array}{r}\text { XSID: CODE } \\
\text { SRD }\end{array}$ & $\begin{array}{l}\text { SRDL } \\
\text { FLEN }\end{array}$ & $\begin{array}{l}\text { LEW } \\
\text { REW }\end{array}$ & $\begin{array}{r}\text { AREA } \\
K\end{array}$ & $\begin{array}{l}\text { VHD } \\
\text { ALPH }\end{array}$ & $\begin{array}{l}\mathrm{HF} \\
\mathrm{HO}\end{array}$ & $\begin{array}{l}\text { EGL } \\
\text { ERR }\end{array}$ & $\begin{array}{r}\text { CRWS } \\
\text { FR\# }\end{array}$ & $\begin{array}{r}Q \\
\text { VEL }\end{array}$ & WSEL \\
\hline סיק & 73 & 0 & 206 & 1.50 & & 498.64 & 492.26 & 2019 & 497 \\
\hline
\end{tabular}




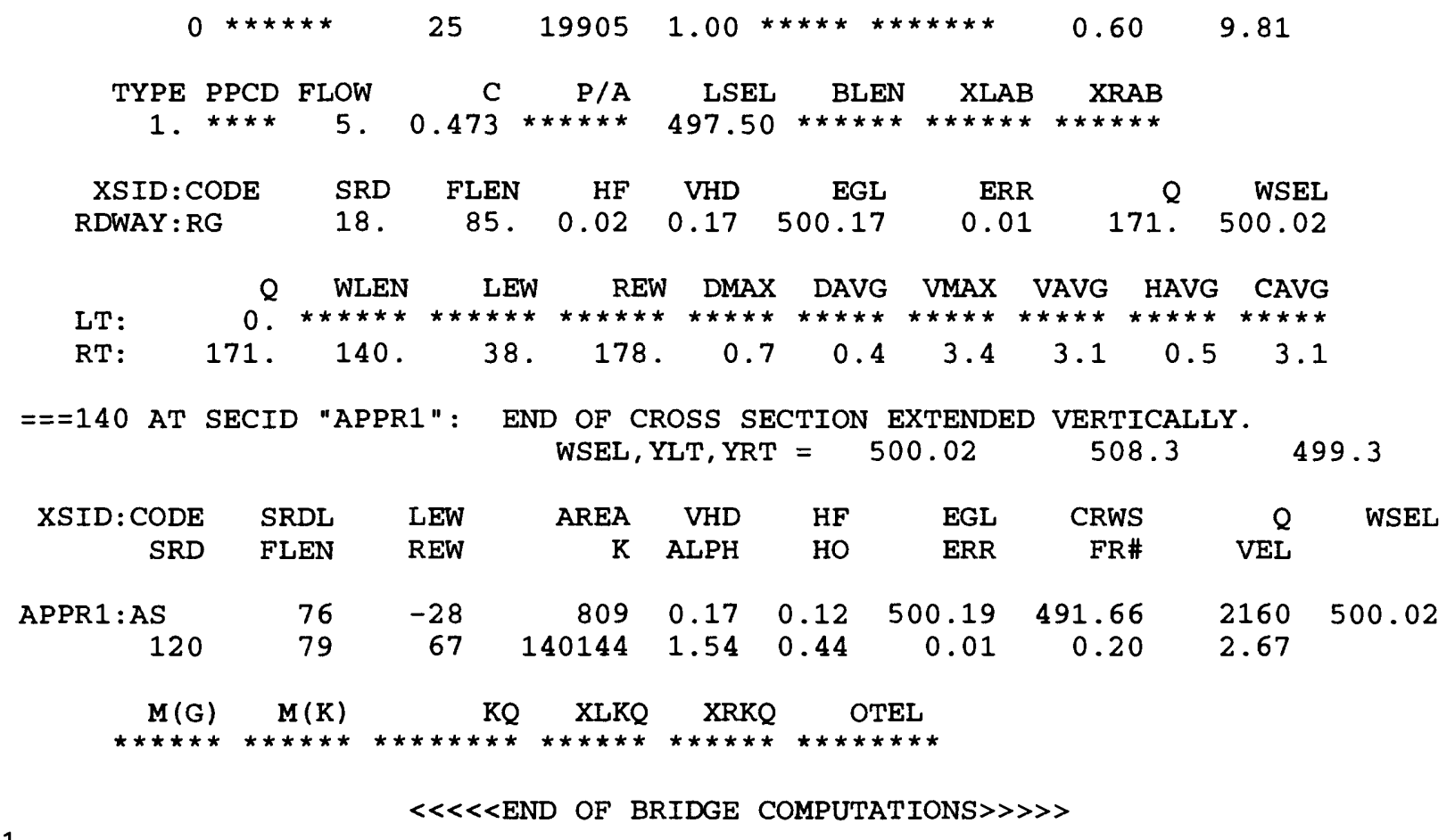

1

FIRST USER DEFINED TABLE.

\begin{tabular}{|c|c|c|c|c|c|c|c|c|}
\hline XSID:CODE & SRD & LEW & REW & $Q$ & $\mathrm{~K}$ & AREA & VEL & WSEL \\
\hline DAMDS : XS & -262 & -30 & 64. & 2160 . & 22707 . & 237 . & 9.10 & 494.66 \\
\hline EXIT2 : XS & -261 & -13 & 60. & 2160 . & 88470 . & 540 & 4.00 & 495.98 \\
\hline EXIT1:XS & -73 & -13 & 60. & 2160 . & 90147 . & 551. & 3.92 & 496.09 \\
\hline FULLV:FV & 0 . & -13 & 60. & 2160 . & 90744 . & 553. & 3.90 & 496.14 \\
\hline BRIDG : BR & 0 . & 0 . & 25 . & 2019. & 19905. & 206 . & 9.81 & 497.1 \\
\hline RDWAY : RG & 18 & $\star \star \star \star \star \star$ & 0 & 171. & 0 . & $\star \star \star \star \star \star$ & 1.00 & 500.02 \\
\hline APPR1: AS & 120 . & -29 & 67. & 2160 . & 140144 . & 809 . & 2.67 & 500.02 \\
\hline
\end{tabular}

XSID: CODE XLKQ XRKQ $\quad$ XQ

SECOND USER DEFINED TABLE.

1

\begin{tabular}{|c|c|c|c|c|c|c|c|c|c|}
\hline XSID: CODE & CRWS & FR\# & YMIN & YMAX & $\mathrm{HF}$ & HO & $\mathrm{VHD}$ & EGL & WSEL \\
\hline DAMDS : XS & 494.66 & 1.13 & 490.58 & $508.27^{\star}$ & & $\star \star \star \star$ & 1.62 & 496.28 & 494 \\
\hline EXIT2 : XS & $\star \star \star$ & 0.28 & 35.15 & 502.88 & 0.00 & 0.00 & & ro & 98 \\
\hline EXIT1:XS & 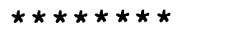 & 0.28 & 5.11 & 2.84 & 0.11 & & & & 09 \\
\hline FULLV : FV & 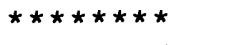 & 0.28 & .12 & 502.85 & 0.04 & & & & 14 \\
\hline BRI & 492.26 & 0.60 & 5.13 & $L 4^{*}$ & $\star \star \star$ & $\star \star \star \star \star \star$ & & 64 & 14 \\
\hline DWAY : RG & & & 9.34 & 508.27 & 0.02 * & * * & 0.17 & 17 & 0.02 \\
\hline APPR1: AS & 491.66 & 0.20 & 486.22 & 508.27 & 0.12 & 0.44 & 0.17 & 500.19 & 500.0 \\
\hline
\end{tabular}

$==015$ WSI IN WRONG FLOW REGIME AT SECID "DAMDS": USED WSI = CRWS. WSI, CRWS $=494.28 \quad 496.11$

\begin{tabular}{|c|c|c|c|c|c|c|c|c|c|}
\hline $\begin{array}{r}\text { XSID: CODE } \\
\text { SRD }\end{array}$ & $\begin{array}{l}\text { SRDL } \\
\text { FLEN }\end{array}$ & $\begin{array}{l}\text { LEW } \\
\text { REW }\end{array}$ & $\begin{array}{r}\text { AREA } \\
\mathrm{K}\end{array}$ & $\begin{array}{l}\text { VHD } \\
\text { ALPH }\end{array}$ & $\begin{array}{l}\text { HF } \\
\text { HO }\end{array}$ & $\begin{array}{l}\text { EGL } \\
\text { ERR }\end{array}$ & $\begin{array}{r}\text { CRWS } \\
\text { FR\# }\end{array}$ & $\begin{array}{r}Q \\
\text { VEL }\end{array}$ & WSEL \\
\hline $\begin{aligned} \text { AMDS : } & X S \\
& -261\end{aligned}$ & 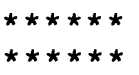 & $\begin{array}{r}-34 \\
78\end{array}$ & $\begin{array}{r}388 \\
40463\end{array}$ & $\begin{array}{l}2.02 \\
1.48\end{array}$ & $\begin{array}{l}\star \star \star \star \star * \\
\star \star \star \star \star *\end{array}$ & $\begin{array}{r}498.13 \\
\star \star \star \star \star \star * \star\end{array}$ & $\begin{array}{r}496.11 \\
1.08\end{array}$ & $\begin{array}{l}3628 \\
9.36\end{array}$ & 1 \\
\hline
\end{tabular}

$===135$ CONVEYANCE RATIO OUTSIDE OF RECOMMENDED LIMITS.

"EXIT2" KRATIO = 2.88 


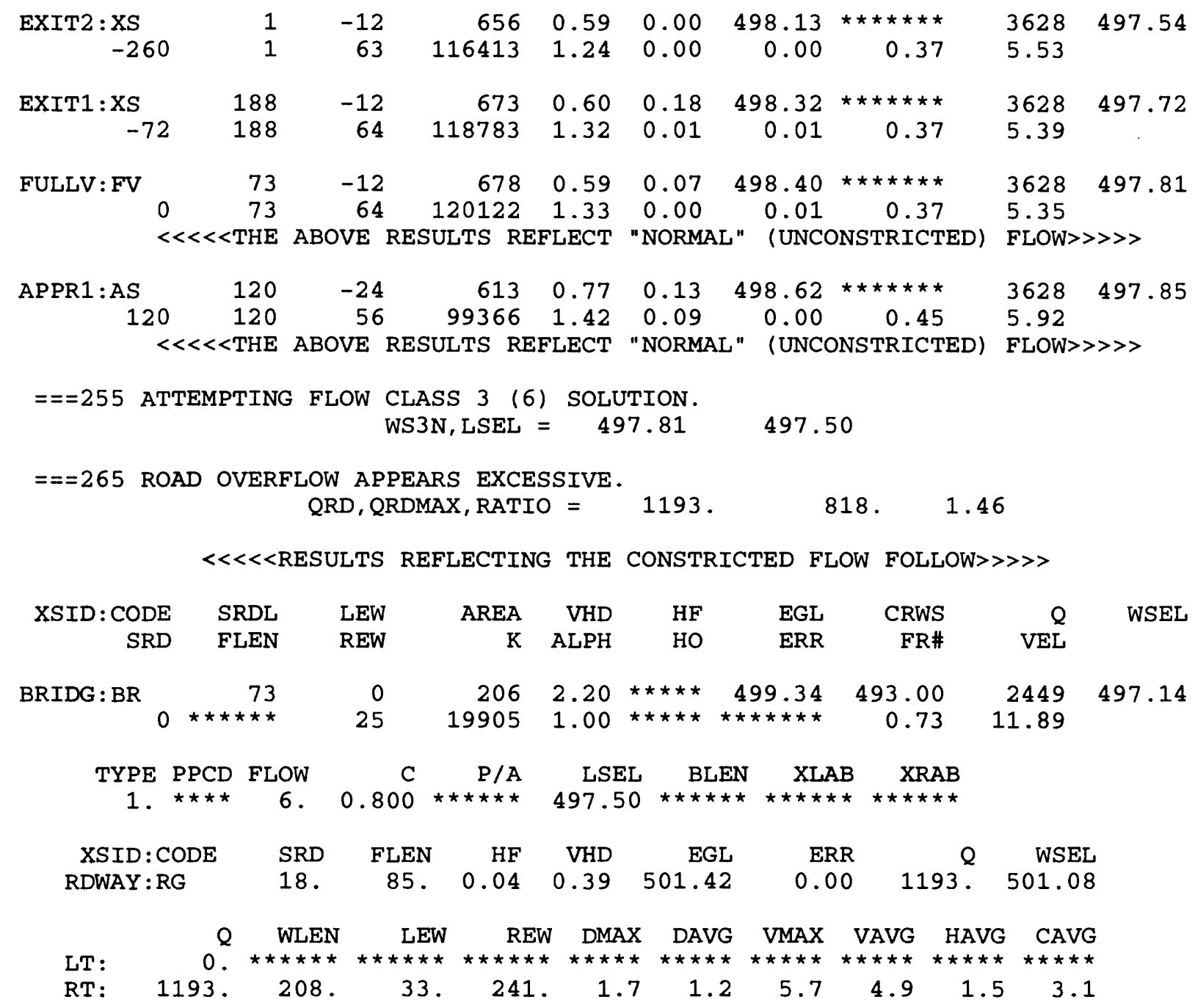

$===140$ AT SECID "APPR1": END OF CROSS SECTION EXTENDED VERTICALLY. WSEL, YLT, YRT $=\begin{array}{ccc}501.08 & 508.3 & 499.3\end{array}$

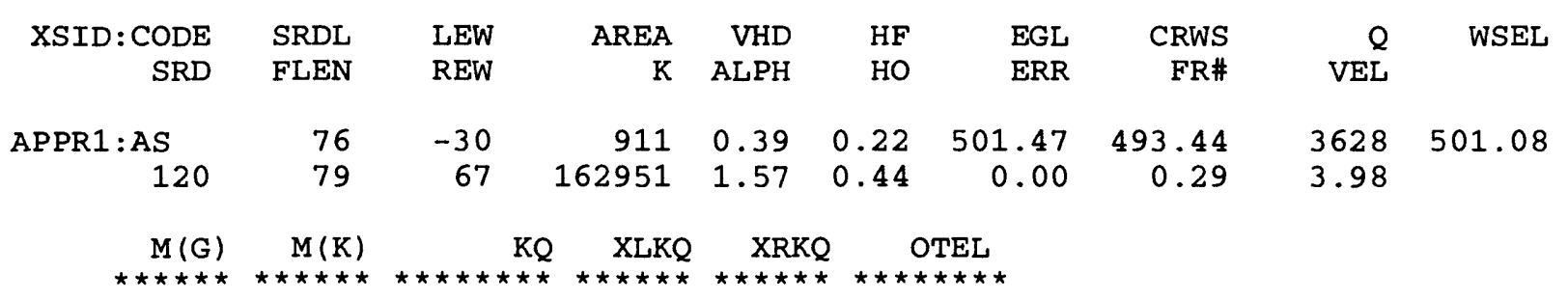

FIRST USER DEFINED TABLE.

$\begin{array}{lrrrrrrrr}\text { XSID : CODE } & \text { SRD } & \text { LEW } & \text { REW } & \text { Q } & \text { K } & \text { AREA } & \text { VEL } & \text { WSEL } \\ \text { DAMDS :XS } & -262 . & -35 . & 78 . & 3628 . & 40463 . & 388 . & 9.36 & 496.11 \\ \text { EXIT2 :XS } & -261 . & -13 . & 63 . & 3628 . & 116413 . & 656 . & 5.53 & 497.54 \\ \text { EXIT1 : XS } & -73 . & -13 . & 64 . & 3628 . & 118783 . & 673 . & 5.39 & 497.72 \\ \text { FULLV : FV } & 0 . & -13 . & 64 . & 3628 . & 120122 . & 678 . & 5.35 & 497.81 \\ \text { BRIDG : BR } & 0 . & 0 . & 25 . & 2449 . & 19905 . & 206 . & 11.89 & 497.14 \\ \text { RDWAY : RG } & 18 . \star \star \star \star \star \star \star & 0 . & 1193 . & 0 . * \star \star \star \star \star \star \star & 1.00 & 501.08 \\ \text { APPR1 : AS } & 120 . & -31 . & 67 . & 3628 . & 162951 . & 911 . & 3.98 & 501.08\end{array}$


SECOND USER DEFINED TABLE.

\begin{tabular}{|c|c|c|c|c|c|c|c|c|c|}
\hline XSID:CODE & CRWS & FR\# & YMIN & YMAX & $\mathrm{HF}$ & HO & VHD & EGL & WSEL \\
\hline DAMDS : XS & 496.11 & 1.08 & 490.58 & $508.27 *$ & $\star \star \star \star \star \star * \star$ & $\star \star \star \star *$ & 2.02 & 498.13 & 496.11 \\
\hline EXIT2:XS & 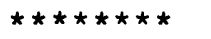 & 0.37 & 485.15 & 502.88 & 0.00 & 0.00 & 0.59 & 498.13 & 497.54 \\
\hline EXIT1:XS & 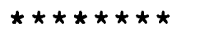 & 0.37 & 485.11 & 502.84 & 0.18 & 0.01 & 0.60 & 498.32 & 497.72 \\
\hline FULLV : FV & 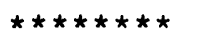 & 0.37 & 485.12 & 502.85 & 0.07 & 0.00 & 0.59 & 498.40 & 497.81 \\
\hline BRIDG : BR & 493.00 & 0.73 & 486.13 & $497.14^{\star}$ & 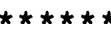 & $\star \star \star \star \star *$ & 2.20 & 499.34 & 497.14 \\
\hline RDWAY : RG & $\star \star \star \star \star \star *$ & * * & 499.34 & 508.27 & $0.04 *$ & 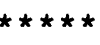 & 0.39 & 501.42 & 501.08 \\
\hline APPR1:AS & 493.44 & 0.29 & 486.22 & 508.27 & 0.22 & 0.44 & 0.39 & 501.47 & 501.08 \\
\hline
\end{tabular}

NORMAL END OF WSPRO EXECUTION. 